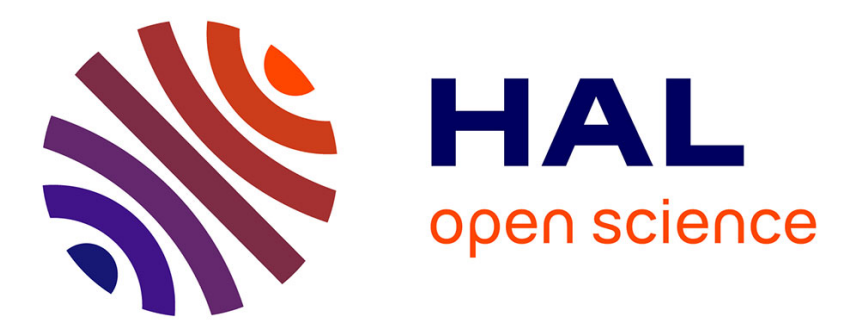

\title{
Domain wall pinning in a circular cross-section wire with modulated diameter
}

\author{
A. de Riz, B. Trapp, J. A. A Fernandez-Roldan, C. Thirion, J.-C. Toussaint, \\ Olivier Fruchart, D. Gusakova
}

\section{- To cite this version:}

A. de Riz, B. Trapp, J. A. A Fernandez-Roldan, C. Thirion, J.-C. Toussaint, et al.. Domain wall pinning in a circular cross-section wire with modulated diameter. Manuel Vazquez. Magnetic Nanoand Microwires, Elsevier, pp.427-453, 2020, 10.1016/b978-0-08-102832-2.00015-3 . hal-02161237

\section{HAL Id: hal-02161237 \\ https://hal.science/hal-02161237}

Submitted on 25 Jun 2019

HAL is a multi-disciplinary open access archive for the deposit and dissemination of scientific research documents, whether they are published or not. The documents may come from teaching and research institutions in France or abroad, or from public or private research centers.
L'archive ouverte pluridisciplinaire HAL, est destinée au dépôt et à la diffusion de documents scientifiques de niveau recherche, publiés ou non, émanant des établissements d'enseignement et de recherche français ou étrangers, des laboratoires publics ou privés. 


\section{Domain wall pinning in a circular cross-section wire with modulated diameter}

A. De Riz ${ }^{1}$, B. Trapp 2 , J. A. Fernandez-Roldan ${ }^{3}$, Ch. Thirion ${ }^{2}$,

J.-Ch. Toussaint ${ }^{2}$, O. Fruchart ${ }^{1}$, and D. Gusakova ${ }^{1}$

${ }^{1}$ Univ. Grenoble Alpes, CNRS, CEA, Spintec, F-38000, Grenoble, France

${ }^{2}$ Univ. Grenoble Alpes, CNRS, Institut NEEL, F-38000, Grenoble

${ }^{3}$ Institute of Materials Science of Madrid, CSIC, 28049, Madrid, Spain

June 20, 2019

\section{Contents}

1 Introduction 3

1.1 Fundamental and technological motivations for domain wall pinning. . . . . . . . . . . . . . . 3

1.2 Types of pinning for nanowires . . . . . . . . . . 4

1.3 Existing theories and experiments . . . . . . . . . 5

2 Theoretical background 6

2.1 Domain walls in cylindrical nanowires . . . . . . . . . . 7

2.2 Geometry of modulation and potential barrier . . . . . . . . 9

2.3 Magnetic charges . . . . . . . . . . . . . . . . . . 11

2.4 Magnetic field generated by the modulation . . . . . . . . 13

2.5 Energy of interaction . . . . . . . . . . . . . 17 
3 Modulation under applied magnetic field 18

3.1 Abrupt modulation . . . . . . . . . . . . . . . . . . . . . 19

3.2 Smooth modulation . . . . . . . . . . . . . . . . . 21

3.3 Protrusion: double abrupt modulation . . . . . . . . . . 24

4 Modulation under applied current $\quad 27$

5 Conclusion and perspective $\quad 30$ 


\section{Abstract}

Domain wall propagation in cylindrical nanowires with modulations of diameter is a key phenomenon to design physics-oriented devices, or a disruptive three-dimensional magnetic memory. This chapter presents a combination of analytical modelling and micromagnetic simulations, with the aim to present a comprehensive panorama of the physics of pinning of domain walls at modulations, when moved under the stimulus of a magnetic field or a spin-polarized current. For the sake of considering simple physics, we consider diameters of a few tens of nanometers at most, and accordingly domain walls of transverse type. Modeling with suitable approximations provides simple scaling laws, while simulations are more accurate, refining the results and defining the range of validity of the models. While pinning increases with the relative change of diameter, a key feature is the much larger efficiency of pinning at an increase of diameter upon considering current rather than field, due to the drastic decrease of current density related to the increase of diameter.

\section{Introduction}

\subsection{Fundamental and technological motivations for do- main wall pinning}

The interest for domain walls in one-dimensional conduits is both for the sake of physics and for technological concepts. As regards physics, considering domain walls in nearly one-dimensional systems allows one to reduce the number of internal degree of freedom to a minimum. In the limit of cylindrical wires with a diameter typically below seven time the dipolar exchange length $l_{\mathrm{ex}}=\sqrt{2 A_{\mathrm{ex}} / \mu_{0} M_{\mathrm{S}}^{2}}$, with $A_{\mathrm{ex}}$ the exchange stiffness and $M_{\mathrm{S}}$ spontaneous magnetization, one can neglect variations of magnetization across the wire section, boiling down the description of the domain wall to a one-dimensional problem [56]. In any case, compared with extended thin films this reduces the possible complexity of the wall, obviously easing the understanding of any phenomena related with domain-wall motion, e.g. precessional dynamics and spin-torques. As regards technology, domain walls have been proposed as means to store[10, 46, 39], transport and process information[7, 8].

It may be desirable to modulate the energy landscape of a domain-wall in such a one-dimensional conduit. This may include potential barriers or 
potential wells. On the fundamental side, such modulations can allow to repeatedly initialize the system with a domain wall at a precise location. This is especially useful to implement time-resolved measurements in a pumpprobe scheme, which requires the averaging of reproducible events, including the preparation of a given type of domain wall[29]. Also, energy barriers may be used to confine a domain wall in a segment of finite length to ease its investigation[16]. On the applied side, a digital memory device requires that bits of information are allocated a specific physical location. Thus, domain walls may be forced to remain in potential wells, or conversely, be separated by energy barriers. Among others, this prevents that successive walls in a conduit merge together, which would induce the loss of information. Also, similar to the argument given above for fundamental devices, defining a precise starting position can be helpful to clock circuits, for instance in the case of logic functions involving several domain walls.

The modulation of potential along has been largely developed and exploited in planar strips based on thin film and lithography technologies. Most are based on the modulation of geometry, which is easily achievable with lithography. This includes notches[60, 29], protrusions[20] or more complex designs such as connection to other magnetic pads[41]. Other means have been demonstrated, such as stray field from neighboring magnetic pads[14] or domain walls[51], ion irradiation[59, 52] or reprogrammable electric-field gating[11].

\subsection{Types of pinning for nanowires}

In the present chapter we focus on cylindrical conduits, which we will call nanowires. Magnetic nanowires have been synthesized routinely for several decades, mostly by e.g. electroplating in polymer or anodized aluminum templates $[27,53,54]$. This synthesis methods presents constraints to design modulations of the potential for domain walls, however also offers opportunities, with respect to flat strips. There exist essentially two designs, which have been developed experimentally and considered theoretically in the past ten years.

The first route for creating a potential landscape, is through the geometry of the wire, involving the longitudinal modulation of the diameter. Indeed, the energy of a domain wall sensitively depends on the wire (local) diameter, involving changes in both exchange and dipolar energy. The most commons means to achieve such a modulation are multistep anodization $[50,54]$ 
or pulsed anodization[40] of aluminum. While the versatility is lower than with lithography for strips, a large variety of designs has been demonstrated. More exotic routes exist, such as pulsed plating followed by etching[22], or the alternation of wire and tubes[48, 45]. The focus of the present work is restricted to the diameter modulation of a plain wire.

The second route for creating a potential landscape, is through the longitudinal modulation of the material. While this is analogous to strips processed with local irradation or gating, it is more straightforward and versatile to achieve in nanowires, by changing the growth conditions during synthesis. The ways to achieve this are multibath anodization for more versatility, or pulsing the plating potential in a bath with several metal salts, for a faster implementation[24, 17]. Note that one may use various magnetic materials, especially varying the composition of compounds[26], or non-magnetic materials such as $\mathrm{Cu}[17,18]$.

\subsection{Existing theories and experiments}

The one-dimensional landscape model for domain walls is probably one of the earliest problems tackled in magnetism to explain the physics of coercivity, as described by the Becker-Kondorski model[12, 13, 36, 37]. A key conclusion is that while domain walls are found at the bottom of energy wells at rest, the depinning field is associated with local maxima of slope of the potential, themselves coinciding with inflexion points of the potential curve. We will see that this concept is still applicable for the more specific theories developed in our contribution. Later on, the one-dimensional landscape model was use again in specific cases by A. Aharoni and followers, again in the context of the physic of coercivity. Potential wells and steps[4], slopes[3] and others, were introduced and described. These effective models have been made more specific to the geometry of a nanowire, highlighting the local slope

A number of micromagnetic simulations have been made, considering linear modulations[5], sharp single modulations[49], sharp constrictions[22], smooth modulations of various length[26]. However, often the processes of domain-wall nucleation at a wire's end and the process of going through the modulation are not studied separately, thus not well describing the latter. Besides, some detailed models of walls at modulations have been proposed[6], however their complexity does not allow to shed a general picture on the phenomenon of pinning. Overall, the existing literature shows interesting features, however does not provide a comprehensive view. This lack has 
been driven the present work, to deriving simple analytical scaling laws, and compare the field-driven and current-driven cases.

Finally, note that experimental reports of the interaction of domain walls at modulations of diameter are still scarce and incomplete. Letting aside reports of magnetometry of large assemblies of wires still in a matrix, or experiments on single wires, however not separating the physics of nucleation from the one of going through the modulation, only a handful of reports exist of domain-walls in diameter-modulated single wires[15]. These do not provide a comprehensive quantitative picture at present.

(a)

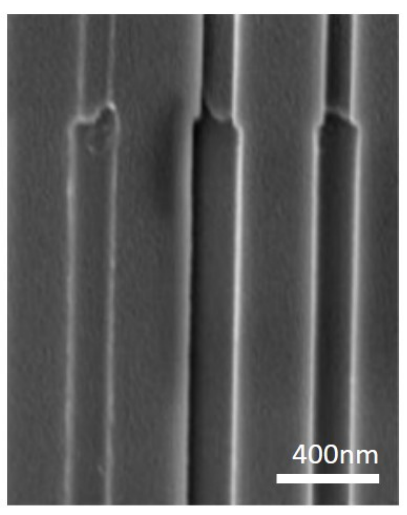

(b)

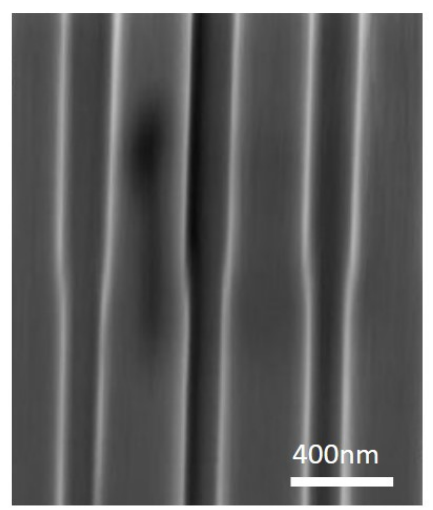

(c)
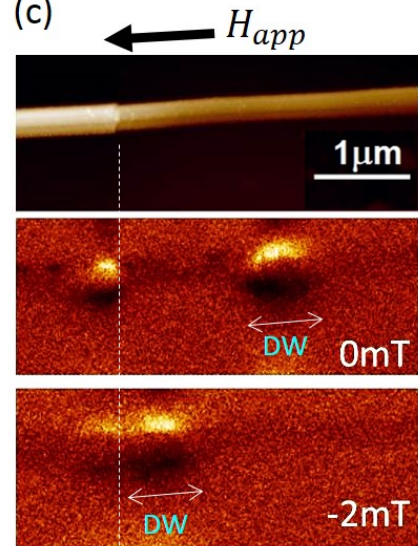

Figure 1: (a) and (b) Scanning electron micrographs illustrating the existence of two different diameter transition geometries in the multisegmented aluminum oxide membranes from [16] and [58]. (c) Topography of isolated multisegmented nanowire and magnetic force microscopy image showing the domain wall displacement after application of $d c$ field [58].

\section{Theoretical background}

The scope of the present section is to recall the basics of nanomagnetism in a circular cross-section nanowire comprising the domain wall, which are relevant for the concepts discussed in the following sections. We start with the energy terms corresponding to ferromagnetic cylindrical nanowires with no modulation, which we will call straight. Then, we introduce the conse- 
quences of the diameter modulations, which imply the existence of an extra magnetic field related to the existence of the magnetic charges.

\subsection{Domain walls in cylindrical nanowires}

(a)

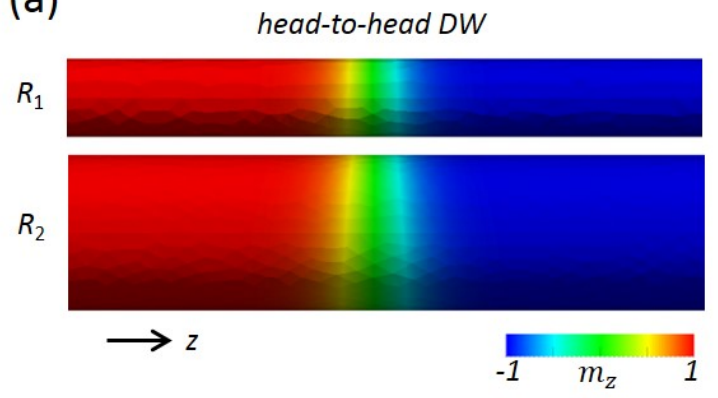

(b)

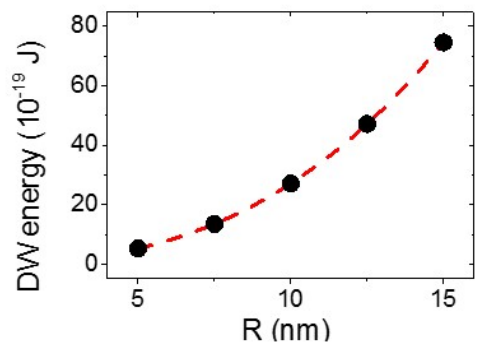

Figure 2: (a) Micromagnetic distribution of longitudinal magnetization for the transverse-like head-to-head domain wall for radius $R_{1}=5 \mathrm{~nm}$ and $R_{2}=10 \mathrm{~nm}$, obtained numerically using equation (1). (b) Simulated domain wall energy vs. diameter, ins straight wire diameter. The dashed curve corresponds is a third-order polynomial fit serving as a guide to the eye.

Domain walls and domains in a ferromagnetic material are usually described within the framework of the micromagnetic theory. First introduced by W.F. Brown [19], it is based on a continuous description of magnetization $\mathbf{M}$ and of all other quantities. The norm of the magnetization vector is assumed to be constant and uniform, so that the local magnetization density can be written as a function of the lateral position $\mathbf{r}$ and of time $t$ as $\mathbf{M}(\mathbf{r}, t)=M_{\mathrm{S}} \mathbf{m}(\mathbf{r}, t)$ with $M_{\mathrm{S}}$ being the spontaneous magnetization. A magnetization distribution can thus be described considering solely the unit vector $\mathbf{m}(\mathbf{r}, t)$, which indicates the local orientation of the magnetization vector. Its time evolution is governed by the Landau-Lifshitz-Gilbert (LLG) equation

$$
\frac{\partial \mathbf{m}}{\partial t}=\gamma_{0} \mathbf{H}_{\mathrm{eff}} \times \mathbf{m}+\alpha \mathbf{m} \times \frac{\partial \mathbf{m}}{\partial t},
$$

where $\gamma_{0}>0$ is the gyromagnetic ratio and $\alpha$ is the phenomenological Gilbert 
damping factor. The effective magnetic field $\mathbf{H}_{\text {eff }}$ is defined as

$$
\mathbf{H}_{\mathrm{eff}}=-\frac{1}{\mu_{0} M_{\mathrm{S}} V} \frac{\delta E}{\delta \mathbf{m}} .
$$

It is related to the system's energy $E$, volume $V$ and magnetic permeability $\mu_{0}$. The first term of the LLG equation reflects the precession of the magnetization vector around the effective field, which may include the internal field contributions as well as the external applied field $\mathbf{H}_{\text {app }}$. Damping of this motion is described in the second term. This equation may be completed by the torque $\mathbf{T}$ produced by the spin-polarized current. However, in this section we do not consider the phenomena related to the spin-polarized current, which are discussed in section 4 .

In most cases of domain-wall motion, it is desirable to minimize extrinsic pinning such as due to spatial fluctuations of magnetic anisotropy, grain boundaries etc. Consequently, here we consider only magnetically-soft materials(such as $\mathrm{Fe}_{20} \mathrm{Ni}_{80}$ ), in particular with no magnetocrystalline anisotropy. Thus, for a straight cylindrical wire[31] under applied magnetic field, the energy $E$ of the system reads:

$$
E=E_{0}+E_{\mathrm{Z}}
$$

where

$$
\begin{gathered}
E_{0}=\frac{\mu_{0}}{2} \int_{V} \mathbf{M} \cdot \mathbf{H}_{\mathrm{d}}(\mathbf{r}) d V+A_{\mathrm{ex}} \int_{V}[\nabla \mathbf{m}(\mathbf{r})]^{2} d V, \\
E_{\mathrm{Z}}=-\mu_{0} M_{\mathrm{S}} \int_{V} \mathbf{m}(\mathbf{r}) \cdot \mathbf{H}(\mathbf{r}) d V .
\end{gathered}
$$

$E_{0}$ is the internal energy. The first term in the equation (4) corresponds to the magnetostatic contribution. The magnitude and orientation of the dipolar field $\mathbf{H}_{\mathrm{d}}$ depend sensitively on the aspect ratio of the ferromagnet. The second term corresponds to the exchange contribution in its continuum form, with the exchange stiffness $A_{\text {ex }}$. When an external field $\mathbf{H}_{\text {app }}$ is applied, its contribution is described as the the Zeeman energy term (5).

For complex magnetic textures or non-trivial geometries, when simplifications of the LLG equation could not be done, the evolution of the magnetic system in time is solved numerically using appropriate micromagnetic codes. In this chapter all numerical simulations have been done using our home-built finite element freeware feeLLGood (Finite Element Landau Lifshitz Gilbert equation Oriented Object Development) $[9,55,1]$. The non-regular finite 
element mesh of feeLLGood accurately describes the cylindrical geometry without creating an artificial numerical roughness at the cylinder surface. Moreover, feeLLGood's parallelized SCALFMM library[2] based on so-called Fast Multipole Method for dipolar field calculation makes it competitive with usually less time consuming finite difference micromagnetic codes.

As we are considering soft magnetic material, the characteristic length scale of choice is the dipolar exchange length $l_{\mathrm{ex}}=\sqrt{2 A_{\mathrm{ex}} / \mu_{0} M_{\mathrm{S}}^{2}}$, resulting from the competition between exchange and magnetostatic energy contributions. Magnetization tends to be rather uniform over distances smaller than $l_{\text {ex }}$, while it may rotate at larger scales under the influence of boundary conditions or dipolar energy. In cylindrical nanowires, depending on the wire diameter so far two different domain wall types have been theoretically predicted [33, 31] and experimentally observed [44, 23, 21, 42]. Moderate wire diameters $\left(D<7 l_{\mathrm{ex}}\right)$ considered in this chapter favor the formation of the socalled transverse-like domain wall $[28,47]$. In one dimension its profile along the $z$ axis is well described by $m_{z}=\tanh (z / \Delta)$ and $m_{y}=1 / \cosh (z / \Delta)$, where $\Delta$ is the wall-width parameter. Fully tree-dimensional transverse-like domain wall distributions obtained by solving numerically equation (1) and corresponding energies are depicted in figure 2 .

\subsection{Geometry of modulation and potential barrier}

A modulation of diameter induces a variation of the internal energy of the system, which depends on the longitudinal position of the domain wall. The center of the wall will be named $z_{\mathrm{DW}}$, which does not mean that we are assuming a wall with zero thickness. In the following, we study four types of modulation profiles, which connect a smaller cross-section with radius $R_{1}$, to a larger cross-section with radius $R_{2}$ : abrupt modulation, straight modulation, circular-based and tanh-based modulations, see figure 3(a).

The abrupt modulation is described by the simple step-function

$$
R(z)= \begin{cases}R_{1}, & z<0 \\ R_{2}, & z>0\end{cases}
$$

The straight modulation of with length $\lambda$ corresponds to the linear function

$$
R(z)= \begin{cases}R_{1}, & z<-\lambda / 2 \\ k z+s, & -\lambda / 2<z<\lambda / 2 \\ R_{2}, & z>\lambda / 2\end{cases}
$$


(a)

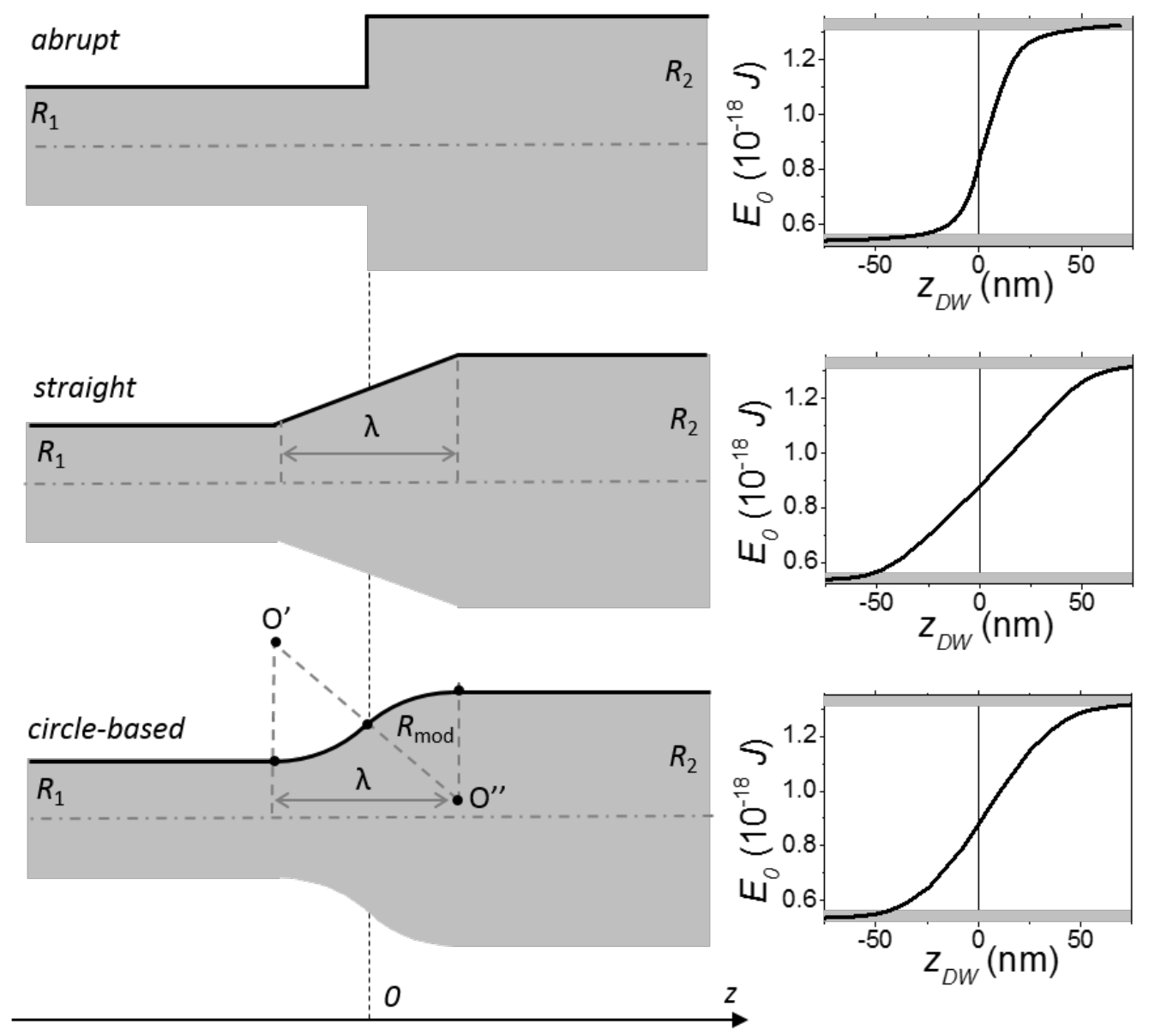

Figure 3: (a) Type of modulation geometry considered and (b) corresponding energy of the domain wall $E_{0}$ vs. its position $z_{\mathrm{DW}}$, from micromagnetic simulations. Parameters used for the energy plots are $R_{1}=5 \mathrm{~nm}, R_{2}=$ $7.5 \mathrm{~nm}, \lambda=100 \mathrm{~nm}$ and $\mu_{0} M_{\mathrm{S}}=5 \mathrm{~T}$. Grey horizontal lines correspond to the energy of a straight wire with $R=5 \mathrm{~nm}$ and $R=7.5 \mathrm{~nm}$.

with $k=\left(R_{2}-R_{1}\right) / \lambda$ and $s=\left(R_{2}+R_{1}\right) / 2$.

The circle-based profile allows for a smooth transition between smaller 
and larger cross-section parts

$$
R(z)= \begin{cases}R_{1}, & z<-\lambda / 2, \\ y_{1}-\sqrt{R_{\text {mod }}^{2}-(z+\lambda / 2)^{2}}, & -\lambda / 2<z<0 \\ y_{2}+\sqrt{R_{\text {mod }}^{2}-(z-\lambda / 2)^{2}}, & 0<z<\lambda / 2 \\ R_{2}, & z>\lambda / 2\end{cases}
$$

with $R_{\text {mod }}=\left[\left(R_{2}-R_{1}\right)^{2}+\lambda^{2}\right] /\left[4\left(R_{2}-R_{1}\right)\right], y_{1}=\left(R_{2}^{2}+2 R_{1} R_{2}-3 R_{1}^{2}+\right.$ $\left.\lambda^{2}\right) /\left[4\left(R_{2}-R_{1}\right)\right]$ and $y_{2}=\left(3 R_{2}^{2}-2 R_{1} R_{2}-R_{1}^{2}-\lambda^{2}\right) /\left[4\left(R_{2}-R_{1}\right)\right]$. Is has been used in subsection 3.2 and in section 4 for the micromagnetic simulations. For the analytic calculations, the circle-based wire profile was approximately replaced by the tanh-based profile

$$
R(z)=\left[R_{1}+R_{2}+\left(R_{2}-R_{1}\right) \tanh (4 z / \lambda)\right] / 2 .
$$

This is an analytic differentiable function, which approximates well the circlebased profile in the case of the gently sloping modulations studied in 3.2 and 4. For the gently sloping modulation with $\left(R_{2}-R_{1}\right)<<\lambda$ the relative error made by tanh-based shape approximation instead of circular-based profile is less than 10 percents.

To illustrate the energy modification, in figure $3(\mathrm{~b})$ we plotted the internal energy $E_{0}$ as a function of the position of the domain wall. These curves were obtained by solving the LLG equation (1) numerically for domain walls drifting freely from the broader part toward the thinner part of the wire in the absence of any driving force. In that case we used $\alpha=1$, to approach a quasistatic situation. The energy of the domain wall is smaller in the thinner part of the wire. Handwavingly, this makes sense as the area and thus the volume of the domain wall, as well as its total magnetic charge and hence the dipolar energy, scale with the wire cross-section. Far from the modulation the value of $E_{0}$ recovers the value of energy of a straight wire, as depicted by horizontal grey lines. The width of the transition between the lower and upper values of $E_{0}$, corresponds approximately to the modulations length $\lambda$.

\subsection{Magnetic charges}

By analogy with electrostatics based on Maxwell's equations, the magnetic volume and surface charges $\rho_{\mathrm{m}}=-M_{\mathrm{S}} \nabla \mathbf{m}$ and $\sigma_{\mathrm{m}}=M_{\mathrm{S}}(\mathbf{n} \cdot \mathbf{m})$, may be introduced as a source of the dipolar field $\mathbf{H}_{\mathrm{d}}[32,34]$, where $\mathbf{n}$ is the outwardpointing unit vector normal to the system surface. The expression for the 
(a)

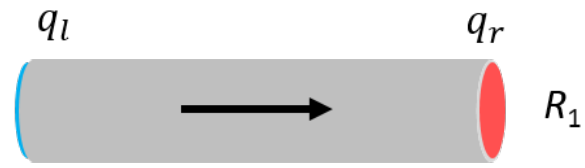

(b)

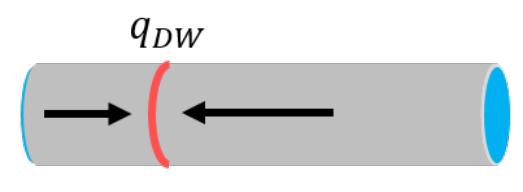

(c)

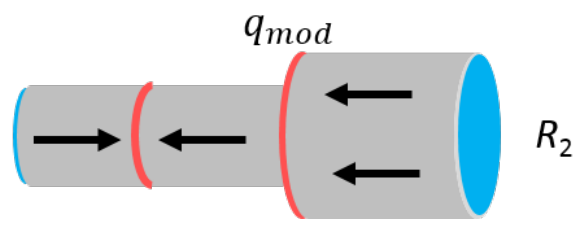

Figure 4: Schematics of magnetic charges distribution in (a) uniformly magnetized cylindrical wire, (b) cylindrical wire with head-to-head domain wall and (c) modulated diameter nanowire with head-to-head domain wall placed in the thinner part. Red color corresponds to the positive magnetic charge and blue one to the negative magnetic charge.

dipolar field reads:

$$
\mathbf{H}_{\mathrm{d}}(\mathbf{r})=\int \frac{\rho_{\mathrm{m}}\left(\mathbf{r}^{\prime}\right)\left(\mathbf{r}-\mathbf{r}^{\prime}\right)}{4 \pi\left|\mathbf{r}-\mathbf{r}^{\prime}\right|^{3}} d^{3} \mathbf{r}^{\prime}+\oint \frac{\sigma_{\mathrm{m}}\left(\mathbf{r}^{\prime}\right)\left(\mathbf{r}-\mathbf{r}^{\prime}\right)}{4 \pi\left|\mathbf{r}-\mathbf{r}^{\prime}\right|^{3}} d S .
$$

In the case of the uniformly magnetized straight cylindrical wire (figure 4 ), each of the wire's end posses the total magnetic charge $q_{1}= \pm \pi M_{\mathrm{S}} R_{1}^{2}$. The total charge is an invariant, whose conservation imposes that the headto-head domain wall in a cylindrical nanowire bears a total magnetic charge $q_{\mathrm{DW}}=2 \pi M_{\mathrm{S}} R_{1}^{2}$. In the case of a modulation of diameter, the two end charges are different: $q_{1}= \pm \pi M_{\mathrm{S}} R_{1}^{2}$ and $q_{2}= \pm \pi M_{\mathrm{S}} R_{2}^{2}$. Considering for instance the case where the domain wall is clearly in the smaller-diameter part, $q_{\mathrm{DW}}=2 \pi M_{\mathrm{S}} R_{1}^{2}$, and a charge is associated with the modulation: $q_{\text {mod }}=\pi M_{\mathrm{S}}\left(R_{2}^{2}-R_{1}^{2}\right)$ (figure $4(\mathrm{c})$ ). At this stage, we did not discussed which type of charge (volume or surface) contributes to the total charge. In all cases (end, modulation, domain wall), the total charge is distributed both over volume charge density as well as the surface charge density, whose distributions are non-trivial. The micromagnetic distribution of the magnetic potential $\phi_{\mathrm{m}}$ related to the charge distribution $\left(\mathbf{H}_{\mathrm{d}}=-\nabla \phi_{\mathrm{m}}\right)$ illustrates this fact in figure 5. Most notably, the modulation charge $q_{\bmod }$ gives rise to a 


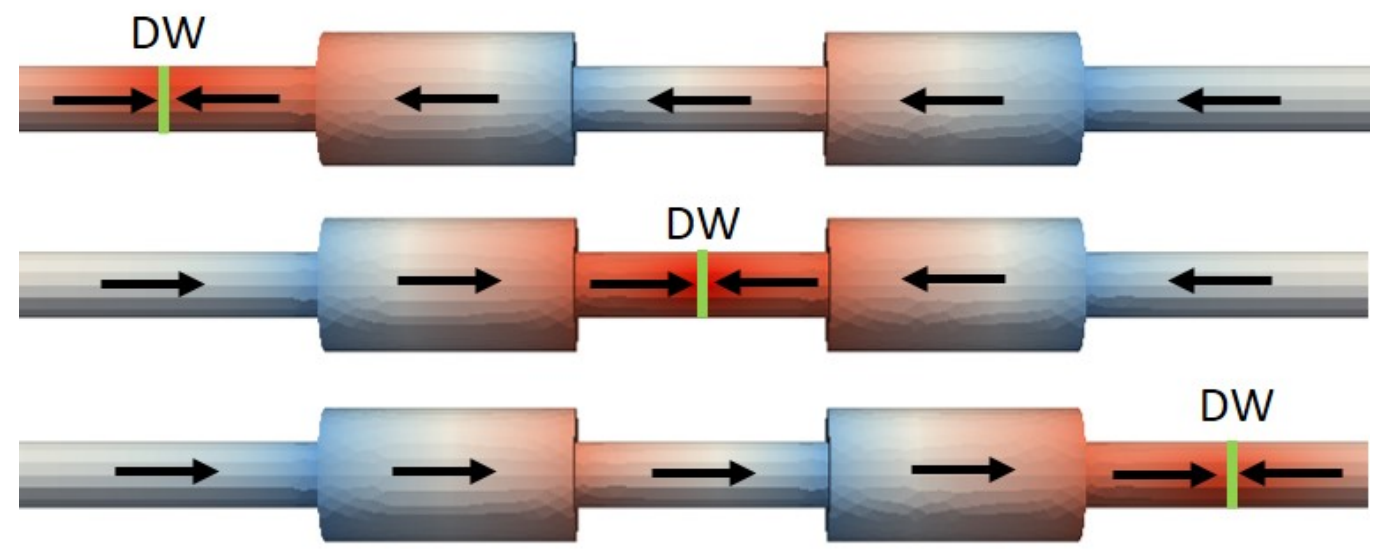

Figure 5: Magnetic potential $\phi_{\mathrm{m}}$ distribution for different positions of the head-to-head domain wall. Red (resp. blue) color corresponds to positive (resp. negative) values of $\phi_{\mathrm{m}}$.

magnetic dipolar field $H_{\text {mod }}$, which we calculate in the next section (subsection 2.4). We show that it tends to move the domain wall away from the modulation.

\subsection{Magnetic field generated by the modulation}

In this section we determine the magnitude and the direction of the magnetic field generated by the modulation of diameter. As regards its contribution of the energy of the system through its interaction with the domain wall, for simplicity we consider its value on the wire axis and at the center of the domain wall $z_{\text {DW }}$.

Following Eq.10, the elementary magnetic field $d \mathbf{H}$ generated by an element of magnetic charge $d q$ at the distance $r$ reads:

$$
d \mathbf{H}=\frac{d q}{4 \pi r^{2}} \frac{\mathbf{r}}{r} .
$$

For the axisymmetrical charge distribution, which is the case here, the resulting magnetic field generated by the whole modulation, is aligned with the $z$ axis. While the the total charge of the modulation is fixed, its distribution over surface and volume contributions is not straightforward. Thus, some approximation that conserves the total charge of the modulation should be 
made. We assume that the magnetization vector in the modulation is strictly aligned with the $z$ axis at each point. This simplification limits the magnetic charge of the modulation to the surface charge $\sigma_{\mathrm{m}}$ only, while volume charges are zero. The surface charge approximation allows us to estimate the amplitude of the magnetic field generated by the modulation analytically in some specific cases.
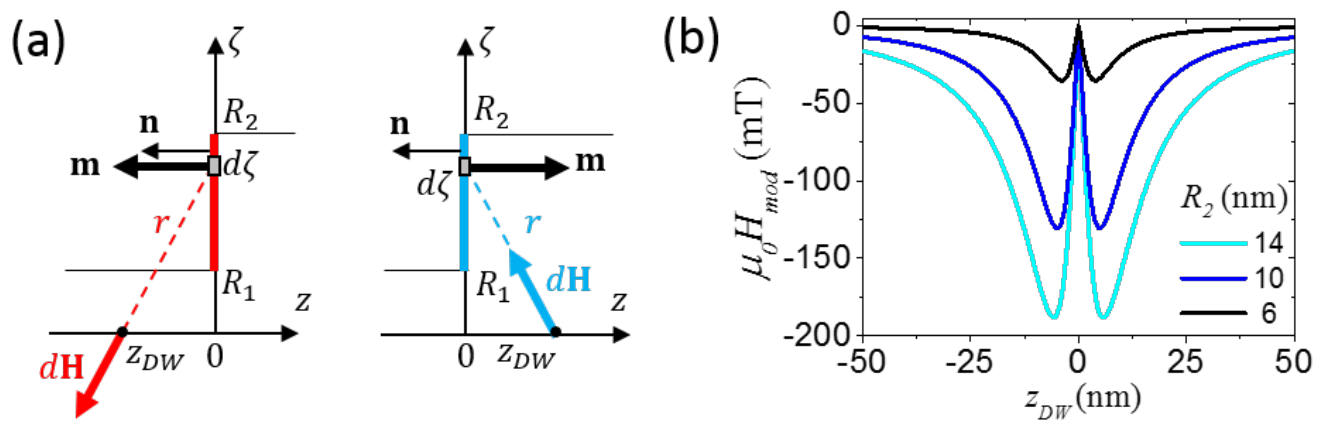

Figure 6: (a) Sketch of the magnetic field generated by the elements of the magnetically charged axisymmetric surface in the presence of the head-tohead domain wall at the position $z_{\text {DW }}$ for abrupt modulation. Red color corresponds to the positive surface charge and blue color to the negative one. (b) Magnetic field generated by the abrupt modulation $\mu_{0} H_{\text {mod }}$ vs. domain wall position $z_{\mathrm{DW}}$ for several values of $R_{2}$. Parameters used for this plot are $R_{1}=5 \mathrm{~nm}$ and $\mu_{0} M_{\mathrm{S}}=1 \mathrm{~T}$.

Abrupt modulation. Besides being close to applicable in some experimental cases, the abrupt modulation is a text-book case, from which the general features of the impact of a modulation on domain-wall motion can be easily illustrated.

With the above assumtions, the abrupt modulation is described by Eq.(6). It is charged positively when the head-to-head domain wall is to the left of the modulation, and negatively when it is to the right of the modulation [Figure 6(a)]. The elementary magnetic field generated by the element $d S$ of the charged surface at the domain wall position $z_{\mathrm{DW}}$, and projected on the $z$ axis, reads:

$$
d H_{z}\left(z_{\mathrm{DW}}\right)=-\frac{\left|z_{\mathrm{DW}}\right|}{r} \cdot \frac{M_{\mathrm{S}} R d R}{2 r^{2}} .
$$

Here $d q=\sigma d S, \sigma= \pm M_{S}(\mathbf{n} \cdot \mathbf{m}), d S=2 \pi R d R, \mathbf{r} / r=\mp 1, d H_{z}=$ 
$d H\left|z_{\mathrm{DW}}\right| / r$ and $r^{2}=z_{\mathrm{DW}}^{2}+R^{2}$. The summation of all contributions from element charges over the entire charged surface gives:

$$
H_{z}\left(z_{\mathrm{DW}}\right)=-\frac{M_{\mathrm{S}}}{2} \int_{R_{1}}^{R_{2}} \frac{\left|z_{\mathrm{DW}}\right| R d R}{\left(R^{2}+z_{\mathrm{DW}}^{2}\right)^{3 / 2}} .
$$

Upon integration, we obtain the magnetic field $H_{\text {mod }} \equiv H_{z}\left(z_{D W}\right)$ generated by the modulation at the center of the head-to-head domain wall

$$
H_{\text {mod }}\left(z_{\mathrm{DW}}\right)=-\frac{M_{\mathrm{S}}\left|z_{\mathrm{DW}}\right|}{2}\left(\frac{1}{\sqrt{R_{2}^{2}+z_{\mathrm{DW}}^{2}}}-\frac{1}{\sqrt{R_{1}^{2}+z_{\mathrm{DW}}^{2}}}\right),
$$

which is plotted in Figure $6(\mathrm{~b})$. The $H_{\text {mod }}$ always opposes the head-to-head domain wall movement to the right, being negative for all $z_{\mathrm{DW}}$. In other words, the charges at the modulation tend to favor motion towards the part with smaller radius, similar to the energy of the domain wall itself.

(a)

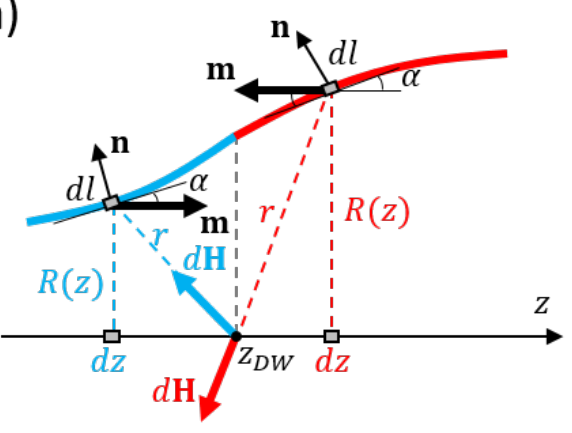

(b)

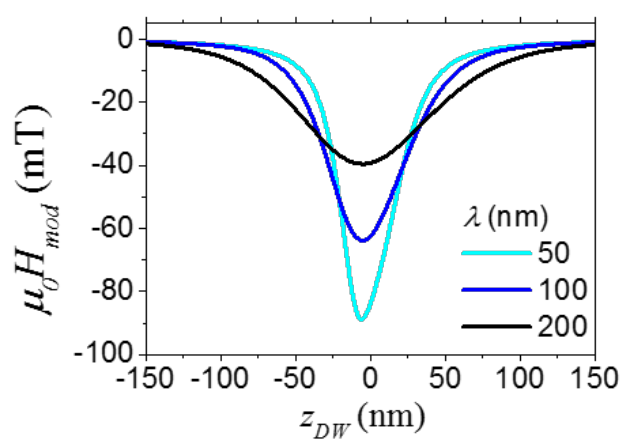

Figure 7: (a) Sketch of the magnetic field generated by the elements of the magnetically charged axisymmetric surface in the presence of the headto-head domain wall at the position $z_{\mathrm{DW}}$ for the modulation of arbitrary profile given by the continuous function $R(z)$. Red color corresponds to the positive surface charge and blue color to the negative one. (b) Magnetic field generated by the tanh-based profile modulation of the length $\lambda$ vs. domain wall position $z_{D W}$ for several values of $\lambda$. Parameters used for this plot are $R_{1}=5 \mathrm{~nm}, R_{2}=10 \mathrm{~nm}$ and $\mu_{0} M_{\mathrm{S}}=1 \mathrm{~T}$.

Charged surface with arbitrary profile. While the main physics is captured by the abrupt modulation, it is associated with an unphysical cusp of $H_{\text {mod }}$ 
at the very center of the modulation. Besides, it may not be realistic for slowly-varying modulations such as found in some experimental cases. The present paragraph intends to describe such situations. Following the same method and assuming only surface charges, let us calculate the magnetic field generated by the modulation with an arbitrary profile given by the continuous function $R(z)$. As shown in Figure 7(a) the corresponding modulation surface is charged positively to the right of the head-to-head domain wall and negatively to the left of it. We may assume a stepwise jump of surface charges across the domain-wall, in the case of gentle modulations. The summation of all contributions from the entire charged surface reads:

$$
H_{\text {mod }}\left(z_{\mathrm{DW}}\right)=-\frac{M_{\mathrm{S}}}{2} \int_{-\infty}^{\infty} \frac{\left|z_{\mathrm{DW}}-z\right| R(z) R^{\prime}(z) d z}{\left[\left(z_{\mathrm{DW}}-z\right)^{2}+R^{2}(z)\right]^{3 / 2}},
$$

This is derived from $\sigma_{\mathrm{m}}=\mp M_{\mathrm{S}} \sin (\alpha), d S=2 \pi R(z) d l, d l=d z / \cos (\alpha)$, $\tan (\alpha)=R^{\prime}(z)$ the derivative of $R(z), d H_{z}=d H\left|z_{\mathrm{DW}}-z\right| / r$ and $r^{2}=$ $\left(z_{\mathrm{DW}}-z\right)^{2}+R(z)^{2}$.

Figure $7(\mathrm{~b})$ depicts $H_{\text {mod }}$ computed using Eq.15 for a tanh-based profile given by the formula 9 . Similar to the case of abrupt modulation, $H_{\text {mod }}$ opposes the head-to-head domain wall movement to the right. However, there is now no more cusp at $z_{\mathrm{DW}}=0$, and the maximum magnitude of $H_{\text {mod }}$ is now found at the center of the modulation. Note that this maximum decreases sharply with increasing modulation length $\lambda$.
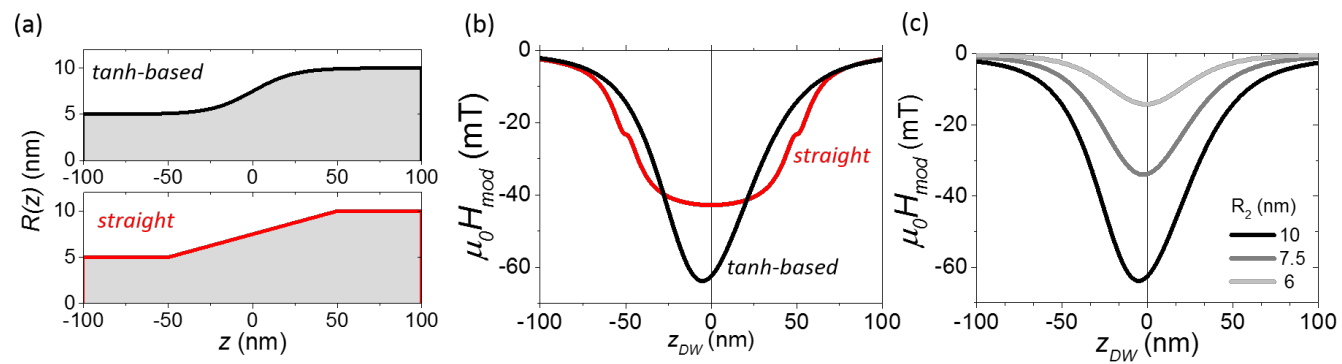

Figure 8: (a) Sketch of the tanh-based and straight profiles with $R_{1}=5 \mathrm{~nm}$, $R_{2}=10 \mathrm{~nm}$ and $\lambda=100 \mathrm{~nm}$. (b) Magnetic field generated by tanh-based and straight modulation profiles illustrated in (a). (c) Magnetic field generated by tanh-based modulation for several values of $R_{2}$. All curves are plotted for $\mu_{0} M_{\mathrm{S}}=1 \mathrm{~T}$. 
Straight modulation. In the case of the straight modulation of three segments [Figure 8(a)] given by the formula (7), the integral in equation (15) should be calculated separately for each segment:

$$
H_{\mathrm{mod}}\left(z_{\mathrm{DW}}\right)=\left\{\begin{array}{l}
\int_{-\lambda / 2}^{\lambda / 2} F\left(z_{\mathrm{DW}}, z\right) d z \\
-\int_{-\lambda / 2}^{z_{\mathrm{DW}}} F\left(z_{\mathrm{DW}}, z\right) d z+\int_{z_{\mathrm{DW}}}^{\lambda / 2} F\left(z_{\mathrm{DW}}, z\right) d z \\
-\int_{-\lambda / 2}^{\lambda / 2} F\left(z_{\mathrm{DW}}, z\right) d z
\end{array}\right.
$$

where

$$
F\left(z_{\mathrm{DW}}, z\right)=\frac{M_{\mathrm{S}}}{2} \frac{\left(z_{\mathrm{DW}}-z\right)(k z+s) k}{\left[\left(z_{\mathrm{DW}}-z\right)^{2}+(k z+s)^{2}\right]^{3 / 2}} .
$$

For very large $\lambda$ we may roughly estimate $H_{\text {mod }}$ considering $k z$ negligible in comparison to $s$. This gives the analytic expression for the field generated by the straight modulation in the following form

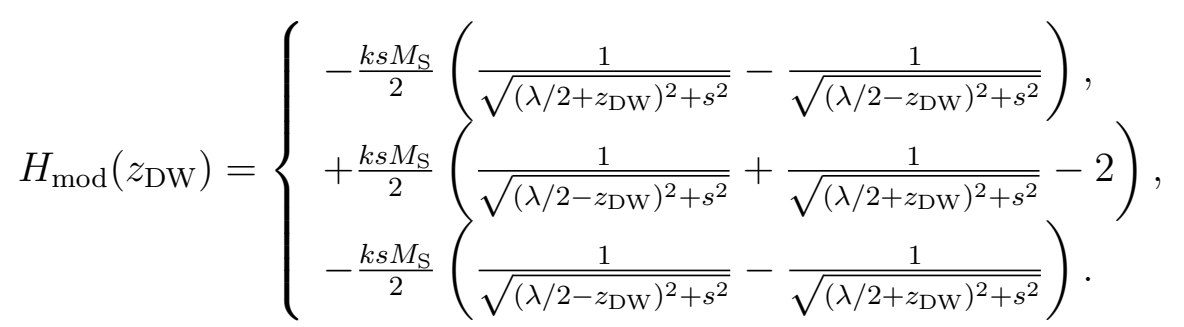

Figure 8 compares $H_{\text {mod }}$ calculated for a straight modulation and a tanhbased profile.

\subsection{Energy of interaction}

In addition to the local terms [Eqs. (4),(5)] describing the domain wall behavior within the straight cylindrical wire, the energy of interaction of the domain wall with modulation charges $E_{\text {mod }}$ must be considered. This is an extra contribution to the internal energy, while the domain wall moves through the modulation, however also at longer range. The total energy now reads:

$$
E_{\text {tot }}=E_{0}+E_{\mathrm{Z}}+E_{\text {mod }} .
$$

The derivative of energy with respect to the wall position, can be written under the form of an effective field. The one associated with the supplementary energy term $E_{\text {mod }}$ is reads, for an axisymmetrical wire: 


$$
\frac{\partial E_{\mathrm{mod}}}{\partial z_{\mathrm{DW}}}=-\mu_{0} q_{\mathrm{DW}} H_{\mathrm{mod}}\left(z_{\mathrm{DW}}\right) .
$$

It is unlikely that $E_{\text {mod }}$ has an analytic expression in the case of an arbitrary modulation profile and arbitrary domain wall profile. In contrast, the field distribution $H_{\text {mod }}\left(z_{\text {DW }}\right)$ can be derived analytically by making some assumptions, as shown in subsection 2.4. Besides, the $z$-derivative of energy may be sufficient, for example, to calculate the domain wall depinning field. In this case we do not need the energy $E_{\text {mod }}$ expression but only its derivative, as the minimization of the total energy gives the domain wall pinned position.

\section{Modulation under applied magnetic field}

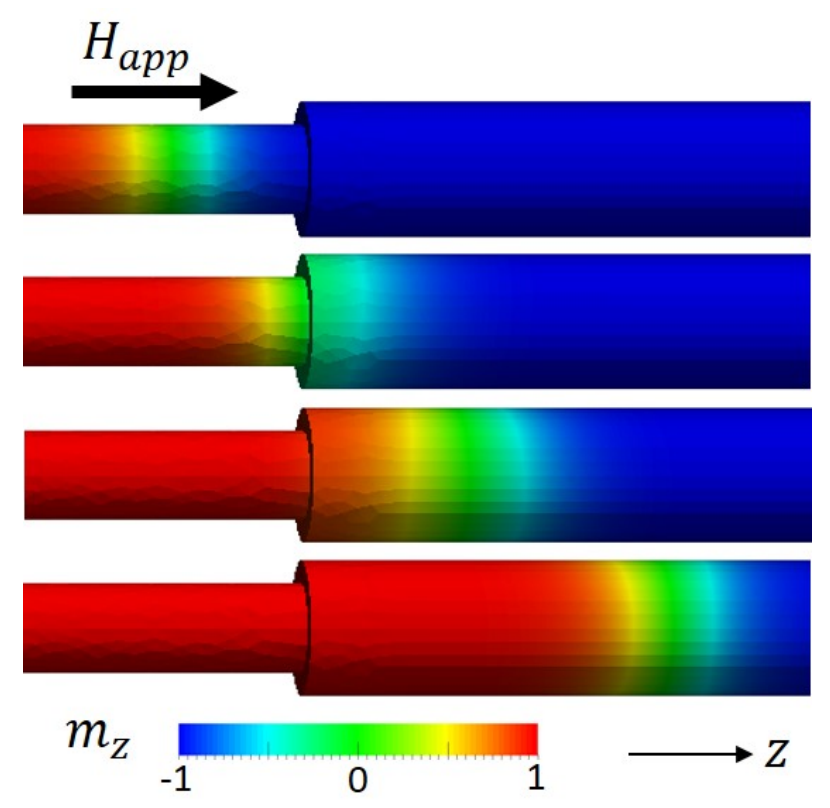

Figure 9: Head-to-head domain wall displacement under the applied magnetic field $H_{\mathrm{app}}$. The color scale bar indicates the longitudinal magnetization $m_{z}$.

In this section we focus on the case of domain wall behavior under a magnetic field applied along the wires axis [Figure 9]. In particular we aim 
to calculate the critical field needed to depin the domain wall. As both the internal and the Zeeman energies are conservative, one may derive the critical field $H_{\text {crit }}$ and corresponding critical domain wall position $z_{\text {crit }}$ on the basis of the position-dependent domain wall energy. In the majority of cases the purely analytical treatment of this problem is tricky or even impossible. For this reason, below we propose an analytical estimation of the $H_{\text {crit }}$ in particular limit cases, which implies a number of simplifying hypothesis. Despite the limitations of the simplified approach, our analytical analysis focuses on the key ingredients and gives a very reasonable estimation of the behavior of the critical depinning field in response to the modulation parameters. The cases for which the assumptions used below are too drastic should be covered by micromagnetic simulations.

\subsection{Abrupt modulation}

(a)

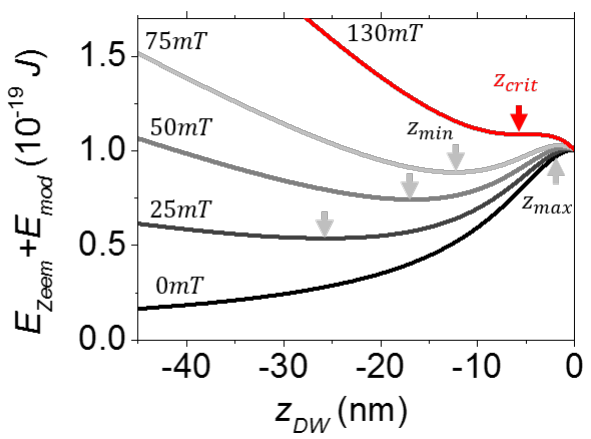

(b)

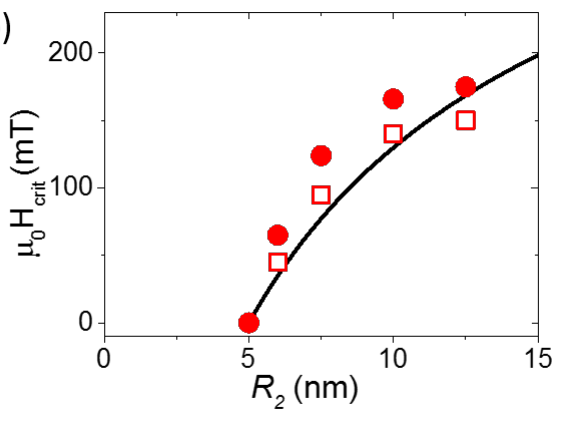

Figure 10: (a) Total energy $E_{\mathrm{Z}}+E_{\text {mod }}$ versus domain wall position $z_{\text {DW }}$ for several values of the applied field and $R_{2}=10 \mathrm{~nm}$. Vertical arrows show the pinned domain wall positions. (b) Critical field value $H_{\text {crit }}$ as a function of the larger radius $R_{2}$. Solid line corresponds to the analytic formula, solid circles and open squares correspond to micromagnetic simulations with $A_{\mathrm{ex}}=$ $1 \cdot 10^{-11} \mathrm{~J} / \mathrm{m}$ and reduced $A_{\mathrm{ex}}=0.25 \cdot 10^{-11} \mathrm{~J} / \mathrm{m}$. All curves are plotted for $\mu_{0} M_{\mathrm{S}}=1 \mathrm{~T}$ and $R_{1}=5 \mathrm{~nm}$.

In this subsection we estimate the critical applied field $H_{\text {crit }}$ needed to depin the domain wall in a wire with an abrupt modulation of diameter, described by equation (6) and visualized in figure 9 . The wire axis was taken as the $z$ direction. The modulation was centered at $z=0$ and $L$ is the 
total length of the wire. The head-to-head domain wall was prepared in the narrow section of the wire, and driven toward the larger section by applying a magnetic field.

Micromagnetic simulations suggest that for such modulation the transition between the two energy levels (or potential barrier) is relatively sharp (figure 3(b)). Moreover, magnetization is mostly perpendicular to the modulation surface, which gives the maximum surface charge $\sigma_{\mathrm{m}}=M_{\mathrm{S}}(\mathbf{m} \cdot \mathbf{n})$ and thus generates the large magnetic field of the modulation (equation (14) and figure 6(b)). In this case it is reasonable to assume that the key ingredient in domain wall pinning is the competition between applied magnetic field $H_{\text {app }}$ and the magnetic field generated by the modulation $H_{\text {mod }}$. Besides, the abrupt jump of diameter, and thus domain wall energy when crossing the modulation, makes that an abrupt jump may not describe all features of the total depinning process. Rather, it is illustrative to describe the long-range competition between the applied field contribution $E_{\mathrm{Z}}=-2 \mu_{0} M_{\mathrm{S}} H_{\mathrm{app}} \pi R_{1}^{2} z_{\mathrm{DW}}+$ Cste and the energy of interaction between domain wall and modulation $E_{\bmod }=-2 \mu_{0} M_{\mathrm{S}} \pi R_{1}^{2} \int H_{\bmod }\left(z_{\mathrm{DW}}, z^{\prime}\right) d z^{\prime}$. This explains the non-monotonic energy profile with domain wall position $z_{\mathrm{DW}}$, as shown in figure 10(a). Note also, that we neglected the inner structure of the domain wall to derive the Zeeman energy, instead we considered the Zeeman energy of two adjacent uniformly magnetized domains on either sides of the domain walls center position, $z_{\mathrm{DW}}$.

The energy derivative $\partial\left(E_{\mathrm{Z}}+E_{\mathrm{mod}}\right) / \partial z_{\mathrm{DW}}=0$ have extrema for $z_{\max }$ and $z_{\min }$. The the latter corresponds to the domain wall pinned position, while the former highlights the top of the energy barrier preventing further motion. Using equations (14), (20) and applied field contribution, we obtain the expression which relates the applied magnetic field to the energy extrema:

$$
H_{\mathrm{app}}=\frac{z_{\mathrm{min}, \max } M_{\mathrm{S}}}{2}\left(\frac{1}{\sqrt{R_{1}^{2}+z_{\min , \max }^{2}}}-\frac{1}{\sqrt{R_{2}^{2}+z_{\min , \max }^{2}}}\right) .
$$

At some critical value of applied field $H_{\text {crit }}$ both extrema $z_{\min }$ and $z_{\max }$ converge into the same point (an inflection point). $z_{\text {crit }}$ may be found using $\partial^{2}\left(E_{\mathrm{Z}}+E_{\text {mod }}\right) / \partial z_{\mathrm{DW}}^{2}=0 . z_{\text {crit }}$ corresponds to the final pinned position of the domain wall:

$$
z_{\text {crit }}=-\frac{R_{1}^{2 / 3} R_{2}^{2 / 3}}{\sqrt{R_{1}^{2 / 3}+R_{2}^{2 / 3}}}
$$


and the corresponding $H_{\text {crit }}$ needed to depin the domain wall reads

$$
H_{\text {crit }}=\frac{M_{\mathrm{S}}}{2}\left(\frac{z_{\text {crit }}}{\sqrt{R_{2}^{2}+z_{\text {crit }}^{2}}}-\frac{z_{\text {crit }}}{\sqrt{R_{1}^{2}+z_{\text {crit }}^{2}}}\right) .
$$

Figure 10(b) compares formula (23) with micromagnetic simulation. This comparison reveals qualitatively and quantitatively similar tendencies. Note that simulations conducted with a value of $A_{\mathrm{ex}}$ reduced in comparison to that of the Permalloy-like material, fits slightly better the analytic results. Is may be explained by the more compact domain wall which probably better suits the model assumptions.

\subsection{Smooth modulation}

In this subsection we estimate the critical applied field $H_{\text {crit }}$ needed to depin the domain wall in a smooth diameter modulation described by equation (9) and schematized in figure 11(a). In practice, the modulation with length $\lambda$ was centered at $z=0$, and $L$ is the total length of the wire. The head-to-head domain wall was prepared in the narrow section of the wire and was driven towards the larger section by applying a magnetic field. To determine the qualitative expression for $H_{\text {crit }}$, we considered the domain wall and Zeeman energies $E_{0}$ and $E_{\mathrm{Z}}$, based on the magnetostatic, exchange and applied field contributions (Eqs.(4),(5)). For simplicity, here we omit the energy of interaction $E_{\text {mod }}$ between the domain wall and the charges of the modulation. It has been shown in [35] for smooth modulations, that the extension of the present model by including the $E_{\text {mod }}$ does not have any qualitative impact, and results only in a slight shift in the total energy minima and maxima. Below, we introduce the approximations that can be used to estimate each energy term. The details of calculation may be found in [35].

For the dipolar energy, we considered that the magnetic charge $q_{\mathrm{DW}}=$ $2 M_{\mathrm{S}} \pi R^{2}$ [38] carried by the head-to-head wall was uniformly distributed within the plain sphere of radius $R$, thus with a magnetic charge density $\rho_{\mathrm{m}}=3 q_{\mathrm{DW}} / 4 \pi R^{3}$. The real distribution of the magnetic charge is much more complex [25],[30]. Nevertheless, our approximation leads to a compact analytical expression for the different energy terms and gives a reasonable order of magnitude. Note that this magnetic charge depends on the domain wall position $z_{D W}$, through $R\left(z_{\mathrm{DW}}\right)$.

By analogy with electrostatics, a dipolar field $H_{\mathrm{d}}$ is generated by the charged plain sphere, with a total magnetostatic contribution $3 \pi \mu_{0} M_{\mathrm{S}}^{2} R^{3} / 5$. 


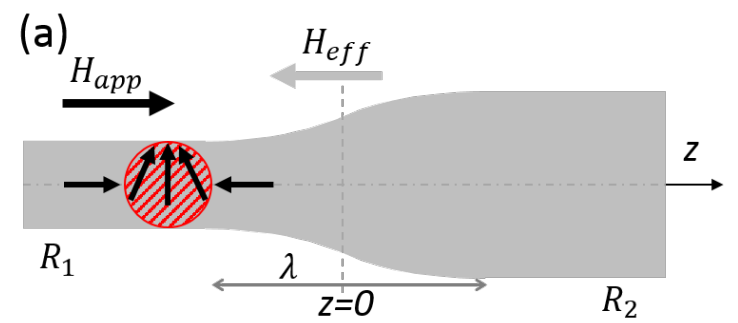

(b)

(c)

(d)
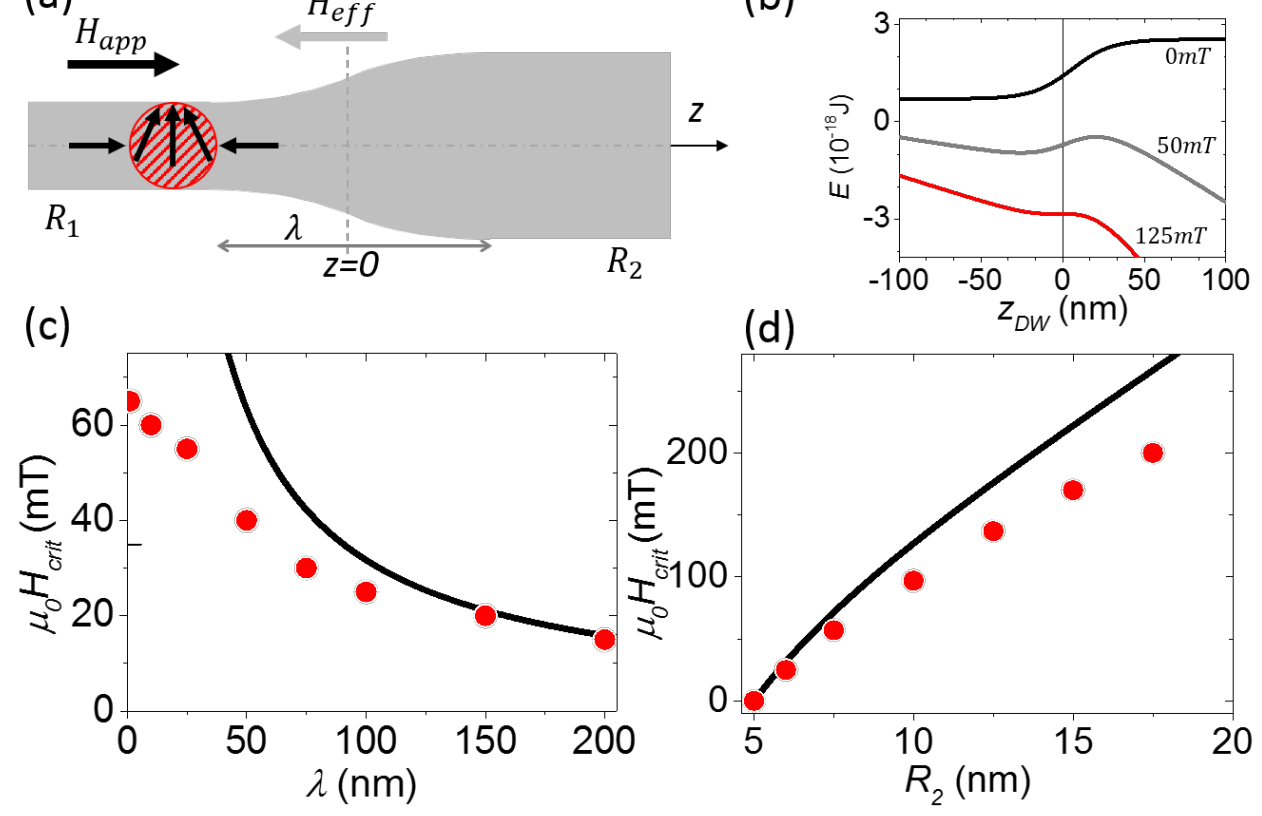

Figure 11: (a)Schematic illustration of the uniformly-charged sphere corresponding to the domain wall. (b) Domain wall energy $E_{0}+E_{\mathrm{Z}}$ as a function of the domain wall position, for several values of applied magnetic field. (c) Critical field $H_{\text {crit }}$ as a function of modulation length $\lambda$ for $R_{2}=6 \mathrm{~nm}$. (d) Critical field $H_{\text {crit }}$ as a function of larger radius $R_{2}$ for $\lambda=100 \mathrm{~nm}$. All curves are plotted for $\mu_{0} M_{\mathrm{S}}=1 \mathrm{~T}$ and $R_{1}=5 \mathrm{~nm}$.

This contribution rapidly grows with the wire radius like $R^{3}$ which is consistent with the micromagnetic simulations of the domain wall energy plotted in figure 2(b) as a function of $R$. The exchange energy contribution can be estimated by applying the one-dimensional spin chain model [32] with slowly varying magnetization. In this case $[\nabla \mathbf{m}(r)]^{2} \approx(\pi / 2 R)^{2}$, so that the total exchange energy contribution equals $A_{\mathrm{ex}} \pi^{3} R / 3$. To estimate the Zeeman energy contribution, we neglected the inner structure of the domain wall and considered the Zeeman energy of two adjacent uniformly magnetized domains located at the domain walls center position, $z_{\mathrm{DW}}$. The domain wall energy 
excluding the integration constant then becomes:

$$
E\left(z_{\mathrm{DW}}\right)=\frac{3 \pi}{5} \mu_{0} M_{\mathrm{S}}^{2} R^{3}\left(z_{\mathrm{DW}}\right)+\frac{A_{\mathrm{ex}}}{3} \pi^{3} R\left(z_{\mathrm{DW}}\right)-2 \mu_{0} M_{\mathrm{S}} H_{\mathrm{app}} \pi \int_{-L / 2}^{z} R^{2}(z) d z
$$

and is depicted in figure 11(b). Note that is it compulsory analytically to consider the finite length of the wire, so that the Zeeman energy is finite.

Both local minima and local maxima are found using energy minimization $\partial E\left(z_{\mathrm{DW}}\right) / \partial z_{\mathrm{DW}}=0$, which gives:

$$
\frac{\partial R\left(z_{\mathrm{DW}}\right)}{\partial z_{\mathrm{DW}}}\left(\frac{18}{5}+\frac{l_{\mathrm{ex}}^{2} \pi^{2}}{3 R^{2}\left(z_{\mathrm{DW}}\right)}\right)-\frac{4 H_{\mathrm{app}}}{M_{\mathrm{S}}}=0
$$

with $l_{\mathrm{ex}}=2 A_{\mathrm{ex}} / \mu_{0} M_{\mathrm{S}}^{2}$. For a tanh-based profile and smooth modulation with $\left(R_{2}-R_{1}\right) /\left(R_{2}+R_{1}\right)<<1$, the coordinates of minimum and maximum of energy reads

$$
z_{\max , \min }= \pm \frac{\lambda}{4} \operatorname{arctanh} \sqrt{1-a H_{\mathrm{app}}}
$$

where $a=\frac{5 \lambda}{9 M_{\mathrm{S}}\left(R_{2}-R_{1}\right)}\left[1+\frac{10 l_{\mathrm{ex}}^{2} \pi^{2}}{27\left(R_{1}+R_{2}\right)^{2}}\right]^{-1}$. The coordinate of the energy minimum $z_{\min }$ corresponds to the domain wall pinned position. It corresponds to an internal effective field $H_{\text {eff }}$ experienced at this point by the center of the domain wall:

$$
H_{\mathrm{eff}}=-H_{\mathrm{app}}=-\left(1-\sqrt{1-H_{\mathrm{app}}}\right) / a .
$$

The domain wall depinning condition, at a given critical applied field value $H_{\text {crit }}$, can be defined as the convergence of two energy extrema at the same point, $z_{\min }=z_{\max }\left(\right.$ red curve in figure $11(\mathrm{~b})$ ). Here we derive $z_{\text {crit }}=0$ for $\left(R_{2}-R_{1}\right) /\left(R_{2}+R_{1}\right)<<1$ (the numerical solution of the equation (25) without this assumption gives slightly different result [35]). The corresponding critical field $H_{\text {crit }}$ reads:

$$
H_{\text {crit }}=\frac{9 M_{\mathrm{S}}\left(R_{2}-R_{1}\right)}{5 \lambda}\left(1+\frac{10 l_{\mathrm{ex}}^{2} \pi^{2}}{27\left(R_{1}+R_{2}\right)^{2}}\right)
$$

and is depicted in figure $11(\mathrm{c})$ and $11(\mathrm{~d})$ as a function of the modulation parameters. The domain wall repulsion from a modulation due to $H_{\text {mod }}$, when not negligible, shifts $H_{\text {crit }}$ towards higher values. Nevertheless, the analytical formula (28) provides a good estimation of $H_{\text {crit }}$ and the relation 
between $H_{\text {crit }}$ and geometric parameters. A key finding is that the critical field is proportional to the slope of the modulation $\left(R_{2}-R_{1}\right) / \lambda$, with a negligibly small exchange correction for small diameters.

The comparison between analytical formula (28) and micromagnetic simulations reveals qualitatively similar tendencies. Moreover, small $R_{2} / R_{1}$ ratios and long $\lambda$, corresponding to gently sloping modulations, ensure the best fit between the simulations and the analytical expression. The cases for which the assumptions used in this model are too drastic should be covered by micromagnetic simulations.

\subsection{Protrusion: double abrupt modulation}

In this subsection we estimate the critical applied field $H_{\text {crit }}$ needed to depin the domain wall in a wire with a protrusion with length $\Lambda$, as schematized in figure 12(a) and given by the formula:

$$
R(z)=\left\{\begin{array}{l}
R_{1}, \quad z<0 \\
R_{2}, \quad 0<z<\Lambda, \\
R_{1}, \quad z>\Lambda
\end{array}\right.
$$

Similar to subsection 3.1, the head-to-head domain wall was prepared in the narrow left section of the wire and driven towards the larger section by applying a magnetic field. To calculate $H_{\text {crit }}$, the assumptions which may be done as well as the procedure to follow, are exactly the same. In order to calculate $H_{\text {crit }}$ for any length $\Lambda$, we should examine the field $H_{\text {mod }}$ created by the charges from both sides of the protrusion. Its expression reads

$$
\begin{array}{r}
H_{\mathrm{mod}}=-\frac{M_{\mathrm{S}}\left|z_{\mathrm{DW}}\right|}{2}\left(\frac{1}{\sqrt{R_{2}^{2}+z_{\mathrm{DW}}^{2}}}-\frac{1}{\sqrt{R_{1}^{2}+z_{\mathrm{DW}}^{2}}}\right) \\
+\frac{M_{\mathrm{S}}\left|z_{\mathrm{DW}}-\Lambda\right|}{2}\left(\frac{1}{\sqrt{R_{2}^{2}+\left(z_{\mathrm{DW}}-\Lambda\right)^{2}}}-\frac{1}{\sqrt{R_{1}^{2}+\left(z_{\mathrm{DW}}-\Lambda\right)^{2}}}\right) .
\end{array}
$$

The magnitude of $H_{\text {mod }}$ is plotted in figure 12(b),(c) and (d) for different values of the protrusion length $\Lambda$. Blue and red lines correspond to the contribution of each side of protrusion separately. The black solid line corresponds to the total $H_{\text {mod }}$. Almost for all values of $\Lambda$ (except for very small $\Lambda$, i.e., for short modulation), $H_{\text {mod }}$ has its deepest minimum to the left of the protrusion (for $z_{\mathrm{DW}}<0$ ). This means, the domain wall should be blocked to the 
(a)

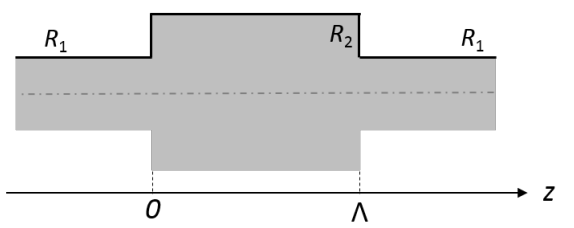

(c)

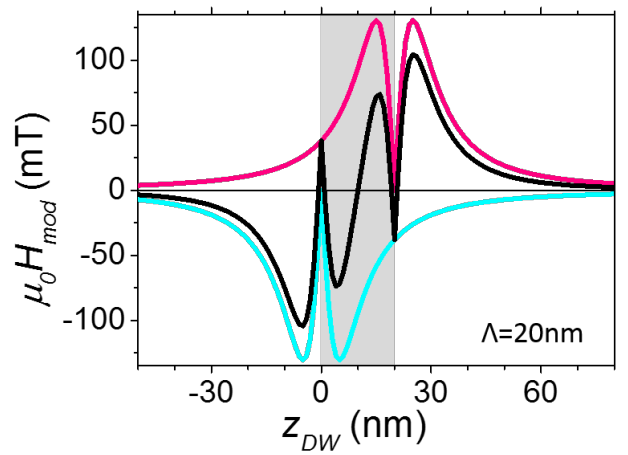

(b)

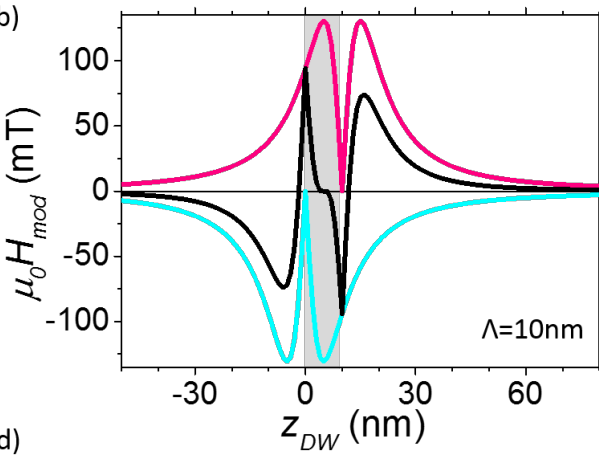

(d)

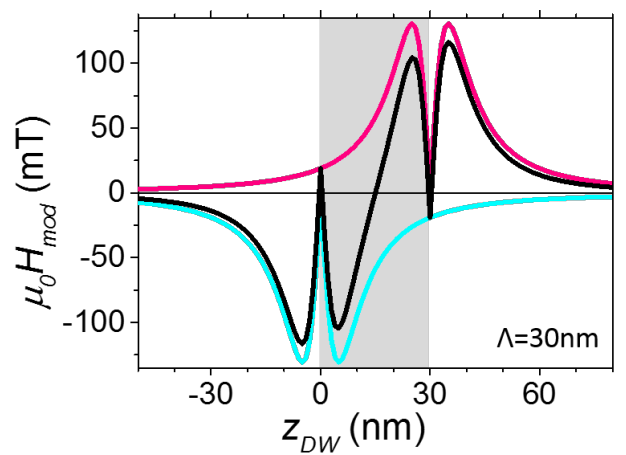

Figure 12: (a) Schematic of the protrusion geometry of the length $\Lambda$ and double abrupt radius modulation between smaller one $R_{1}$ and larger one $R_{2}$. (b),(c) and (d) Analytical curve for the magnetic field generated by a double modulation, for three values of the protrusion length $\Lambda$. Red and blue lines correspond to the contribution of each modulation and black line to the total resulting magnetic field $H_{\text {mod }}$. All curves are plotted for $\mu_{0} M_{\mathrm{S}}=1 \mathrm{~T}$, $R_{1}=5 \mathrm{~nm}$ and $R_{2}=10 \mathrm{~nm}$.

left of the protrusion. For very small $\Lambda$, the situation maybe reversed (figure 12(b)) and the domain wall may be pinned to the right of the protrusion. In order to quantify this phenomenon we followed the same procedure for the energy minimization similar to single abrupt modulation case and solved numerically $\partial^{2}\left(E_{\mathrm{Z}}+E_{\mathrm{mod}}\right) / \partial z_{\mathrm{DW}}^{2}=0$ condition. This numerical solution gives $H_{\text {crit }}$ as a function of protrusion length $\Lambda$, and is plotted in figure 13(a).

This gives rises to two regimes. As expected, for large $\Lambda$ (wide protrusion), the critical field $H_{\text {crit }}$ recovers the single abrupt modulation value. The pinning position is ahead of the protrusion, like for a single increase 
(a)

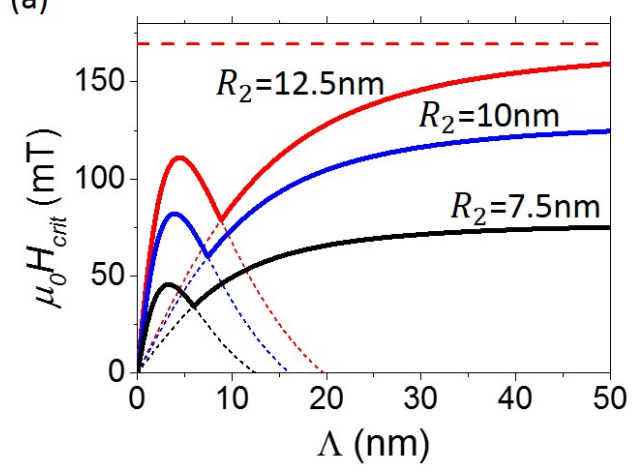

(b)

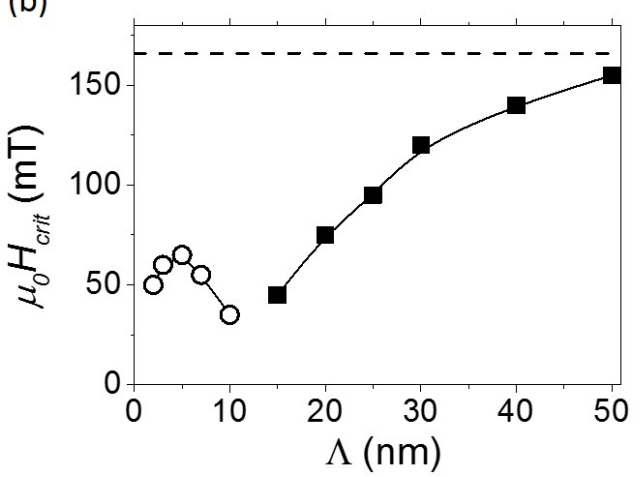

(c)

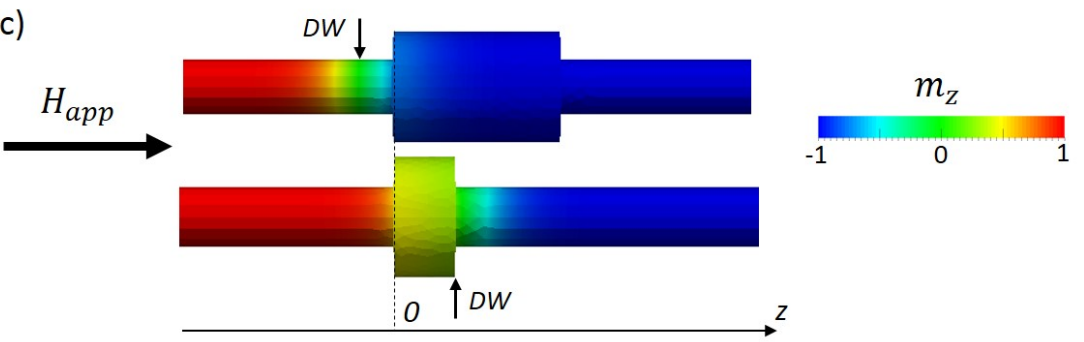

Figure 13: (a) Critical field $H_{\text {crit }}$ as a function of the protrusion length $\Lambda$ for several values of $R_{2}$. Red horizontal dashed line corresponds to the critical field value for $\Lambda=\infty$ (single abrupt modulation) and $R_{2}=12.5 \mathrm{~nm}$. (b) Micromagnetic simulation of the $H_{\text {crit }}$ as a function of the protrusion length $\Lambda$ for $R_{2}=10 \mathrm{~nm}$. The branch with solid squares corresponds to the domain wall pinned to the left of the protrusion. The branch with open circles corresponds to the domain wall pinned to the right of the protrusion. Black horizontal dashed line corresponds to the critical field value for $\Lambda=\infty$ (single abrupt modulation). (c) Domain wall pinned to the left of the protrusion for $\Lambda=30 \mathrm{~nm}$ and domain wall pinned to the right of the protrusion for $\Lambda=10 \mathrm{~nm}$. The color scale bar indicates the longitudinal magnetization $m_{z}$. All graphs are plotted for $\mu_{0} M_{S}=1 \mathrm{~T}$ and $R_{1}=5 \mathrm{~nm}$.

of diameter. Once the domain wall enters the protrusion, propagation proceeds beyond the center of the protrusion as the geometry favors expulsion, adding its effect to the Zeeman energy. For decreasing protrusion lengths the pinning field also decreases. This is related to the partial balance of the 
repulsive charge on the left side of the protrusion and the attractive charge on the right side of the modulation. For very small values of $\Lambda$, the critical field $H_{\text {crit }}$ has non-monotonic behavior, which correspond to the case with the domain wall pinned to the right of the modulation.

However, the model considers a domain wall with zero width, an hypothesis that may be put at stake for a short modulation. Besides, we stressed in section 3.1 that the model with abrupt modulation may not describe accurately the situation when the domain wall enters the modulation. For these reasons, it is important to check the situation with micromagnetic simulations. Surprisingly, these reproduce rather well the tendencies obtained in the simplified model, as shown in figures 12(b) and 12(c). Among others, the existence of two regimes is confirmed. This stresses that care needs to be taken in the analysis of experimental data, for which multiple pinning sites may not results from extrinsic imperfections.

\section{Modulation under applied current}

In this section we describe the domain wall behavior under applied current. For the sake of providing a realistic picture even when crossing the modulation, we immediately jump to the case of the smooth diameter modulation given by the tanh-based profile formula (9) and schematized in figure 14(a). The wire axis is again taken as the $z$ direction. The modulation of the length $\lambda$ is centered at $z=0$ and $L$ is the total length of the wire. The head-tohead domain wall was prepared in the narrow section of the wire and driven towards the larger section by applying a spin-polarized current, with the electrons flowing from the narrow to the broad section. Similar to subsection 3.2, we make several simplifying assumptions and focus on the key ingredients to estimate the critical current needed to depin the domain wall. We then use micromagnetic simulations to refine the analytic picture.

Under the applied spin-polarized current the domain wall motion obeys the LLG equation (1) generalized with the so-called adiabatic and nonadiabatic spin torques [57]

$$
\mathbf{T}=-\frac{P \mu_{\mathrm{B}}}{e M_{\mathrm{S}}}[(\mathbf{J} \cdot \nabla) \mathbf{m}-\beta \mathbf{m} \times(\mathbf{J} \cdot \nabla) \mathbf{m}],
$$

with $P$ the spin-polarization ratio of the current, $\mu_{\mathrm{B}}$ the Bohr magneton, $e$ the (positive) elementary charge, $\beta$ the non-adiabatic coefficient and $\mathbf{J}$ the 
(a)

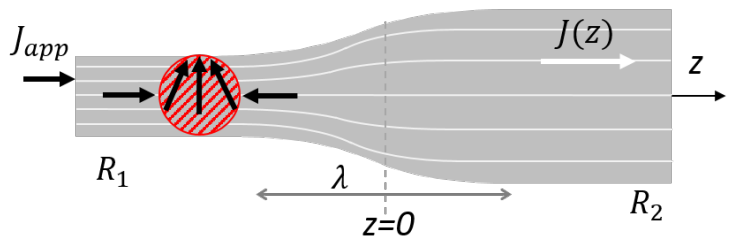

(c)

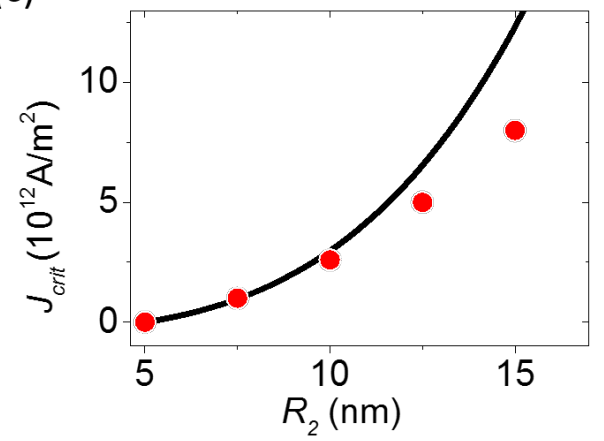

(b)

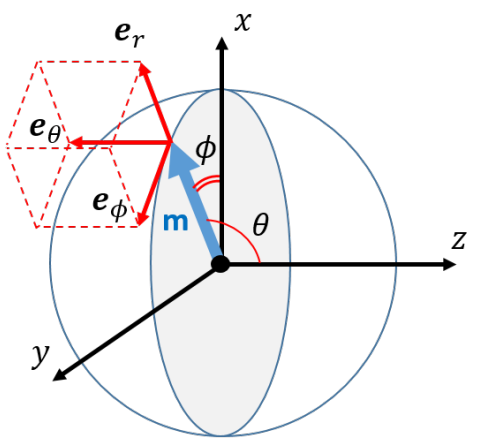

(d)

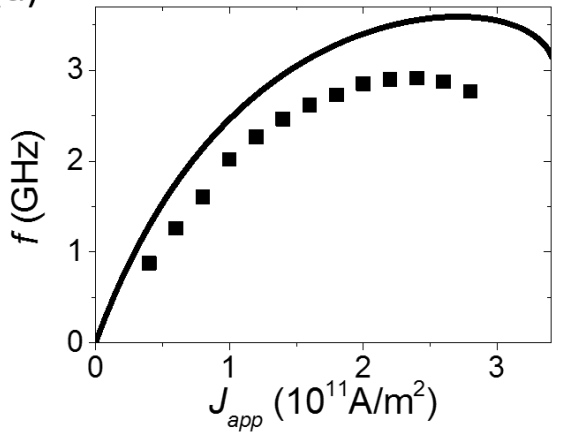

Figure 14: (a)Illustration of the domain wall under applied current in a modulated diameter wire. (b) Spherical coordinate basis $\left\{\mathbf{e}_{r}, \mathbf{e}_{\theta}, \mathbf{e}_{\phi}\right\}$ for magnetization vector $\mathbf{m}$ and Cartesian spatial coordinates $x, y$ and $z$. The magnetization vector is drawn in the particular position corresponding to $\theta=\pi / 2$, so that $\mathbf{e}_{\theta}=-\mathbf{e}_{z}$. (c) Critical current density value $J_{\text {crit }}$ versus the larger radius $R_{2}$. The solid curve corresponds to the equation (35). Points correspond to the micromagnetic simulations. (d) Domain wall rotation frequency $f$ in a pinned state vs. applied current density $J_{\text {app }}$ for $R_{2}=10 \mathrm{~nm}$. The solid curve corresponds to the equation (36). Points correspond to the micromagnetic simulations. Curves (c) and (d) are plotted for $\mu_{0} M_{\mathrm{S}}=1 \mathrm{~T}, R_{1}=5 \mathrm{~nm}$, $\lambda=100 \mathrm{~nm}, \alpha=0.02, \beta=0.04$ and $P=0.7$.

electron current density. It is convenient to express the magnetization vector and the effective field in the spherical coordinates basis $\left\{\mathbf{e}_{r}, \mathbf{e}_{\theta}, \mathbf{e}_{\phi}\right\}$ [figure 14(b)] with $\mathbf{m}=\mathbf{e}_{r}, \mathbf{H}_{\text {eff }}=H_{r} \mathbf{e}_{r}+H_{\theta} \mathbf{e}_{\theta}+H_{\phi} \mathbf{e}_{\phi}$ and $\dot{\mathbf{m}}=\dot{\theta} \mathbf{e}_{\theta}+\sin \theta \dot{\phi} \mathbf{e}_{\phi}$. The circular symmetry of the nanowire leads to the energy rotational invariant, which implies $\partial / \partial \phi=0$ and thus $H_{\phi}=0$. Moreover, for simplicity we 
neglect any azimuth distortion of the domain wall, which corresponds to the 1-dimensional spin chain and implies $\nabla \phi=0$. We name $J_{\text {app }}$ the current density in the narrow part of the wire, and assume that the electron current is parallel to the $z$ axis $\mathbf{J}_{\text {app }}=J_{\text {app }} \mathbf{e}_{z}$ (figure $14(\mathrm{a})$ ). This approximation is suitable for a smooth cross-section. Similarly, the electron current density is considered uniform in a cross-section and is related to the applied current density through $J_{z}(z)=R_{1}^{2} J_{\text {app }} / R^{2}(z)$.

After some algebra the LLG equation (1) augmented by the spin-torque (Eq.(31)) takes the form

$$
\begin{gathered}
\dot{\theta}=\frac{\gamma_{0}}{1+\alpha^{2}} \alpha H_{\theta}-\frac{1+\alpha \beta}{1+\alpha^{2}} \frac{\sin \theta}{\Delta} \frac{P \mu_{\mathrm{B}}}{e M_{\mathrm{S}}} J_{z}(z), \\
\sin \theta \dot{\phi}=-\frac{\gamma_{0}}{1+\alpha^{2}} H_{\theta}+\frac{\beta-\alpha}{1+\alpha^{2}} \frac{\sin \theta}{\Delta} \frac{P \mu_{\mathrm{B}}}{e M_{\mathrm{S}}} J_{z}(z),
\end{gathered}
$$

where $H_{\theta}=-\left(\mu_{0} M_{\mathrm{S}} V\right)^{-1} \delta E / \delta \theta, \dot{\theta}=v \sin \theta / \Delta, v$ is the forward domain wall velocity and $\dot{\phi}=2 \pi f$ is the angular domain wall velocity. Here we applied the useful property of a 1-dimensional domain wall profile $\partial \theta / \partial z=\sin \theta / \Delta$, where $\Delta$ is the wall-width parameter $[31,43]$.

For simplicity in the following we omit the internal domain wall structure and study the behavior of the magnetization vector in its center of the domain wall, where $\theta=\pi / 2$. Let us assume that the domain walls settles at a given position $z_{\mathrm{DW}}$, for a given value of applied current $J_{\text {app }}$. This corresponds to $\dot{\theta}=0$, which from Eq.(32) implies:

$$
H_{\theta}=\frac{P \mu_{\mathrm{B}}}{e M_{\mathrm{S}}} \frac{(1+\alpha \beta) R_{1}^{2} J_{\mathrm{app}}}{\alpha \gamma_{0} R^{2}(z) \Delta} .
$$

In the center of the domain wall, where $\mathbf{e}_{\theta}=-\mathbf{e}_{z}, H_{\theta}$ is parallel to the $z$ axis and is pointed to the negative $z$ direction, so that $H_{\theta}=-H_{\text {eff }}$. From Eq.(3), $H_{\theta}=-H_{0}$ is the internal field, reflecting the $z$-dependence of the domain wall energy, which we calculated at any position in the field-driven model. From this, for a given $J_{\text {app }}$ we can solve Eq.(34) to search for a $z$ value allowing an equilibrium position. Thus, by combining equations (27), (28) and (34), we obtain a relation linking the applied current $\left(J_{\text {app }}\right)$ and the resulting steady-state position of the domain wall $z_{\text {eq }}$. Such a position exists for moderate current, however not for very large current. The cross-over determines the depinning current $J_{\text {crit }}$ in a smoothly-varying modulation: 


$$
J_{\text {crit }}=\frac{9 \alpha \gamma_{0} e M_{\mathrm{S}}^{2}}{20 P \mu_{\mathrm{B}}} \frac{\left(R_{1}+R_{2}\right)^{3}\left(R_{2}-R_{1}\right)}{R_{1}^{2} \lambda}\left(1+\frac{10 l_{\mathrm{ex}}^{2} \pi^{2}}{27\left(R_{1}+R_{2}\right)^{2}}\right) .
$$

Here, similar to subsection 3.2 , we have assumed $z_{\text {crit }} \cong 0$, and $\Delta \cong$ $2 R\left(z_{\text {crit }}\right)=R_{1}+R_{2}$. This law is plotted in figure 14 . If we compare the domain wall behavior under applied field (figure 11(d)) and under applied current (figure 14(c)) in a smooth modulation, there is a major difference in the efficiency of the driving force in both cases. The critical field $H_{\text {crit }}$ is almost linear with growing larger diameter whereas the growth of $J_{\text {crit }}$ follows the power low of $R_{2}$. The reason is the decrease of local current density when the section broadens: not only does the domain wall energy increase, but the efficiency of spin torque decreases. Besides, $J_{\text {crit }}$ is proportional to the domain wall width-parameter which grows in the larger cross-section.

Figure 14(c) compares the analytical solution with micromagnetic simulations. The tendencies are similar, with even an excellent quantitative agreement in the limit of gentle modulation. This validates the model, and the above conclusion.

The model also predicts the frequency of precession of the transverse component of magnetization of the wall, at the pinned position:

$$
f=\frac{P \mu_{\mathrm{B}}}{2 \pi e M_{\mathrm{S}}} \frac{R_{1}^{2} J_{\mathrm{app}}}{\alpha R^{2}(z) \Delta} .
$$

The dominant effect is that of the internal field and not of the non-adiabatic spin torque, resulting in the $1 /(\alpha \Delta)$ coefficient in this equation. This frequency is plotted in figure $14(\mathrm{c})$, for which similar to subsection 3.2 , we estimated the wall-width parameter $\Delta$ as $\Delta \cong 2 R(z)$. Again, an excellent agreement is found with numerical simulation.

\section{Conclusion and perspective}

We have derived analytical models to describe how a magnetic domain wall may go through a modulation of diameter in a cylindrical nanowire, under the stimulus of either a magnetic field or a spin-polarized current. Scaling laws are derived, which may be used to quickly design a modulation to reach specific properties. While the depinning field scales with $M_{\mathrm{S}}$ and the slope of the modulation $\left(R_{2}-R_{1}\right) / \lambda$, the depinning current increases much faster 
with the geometrical strength of the modulation, due to the decrease of local current density, and the increase of wall width. The relevance of these laws are are confirmed by micromagnetic simulations, which reveal an excellent quantitative agreement for smoothly-varying modulations.

The quantitative investigation of experimental domain-wall pinning in modulations of diameter is still in its infancy. It has been carried-out under magnetic field so far, with scattered results, however pointing at the moderate strength of pinning compared with extrinsic pinning on material defects, when considering smoothly-varying modulations. The drastically higher efficiency of pinning under current, raises hope that modulations of diameter can be designed efficiently for spin-torque fundamental or applied devices.

\section{Acknowledgements}

We acknowledge financial support from the French National Research Agency (ANR) (Grant No. JCJC MATEMAC-3D) and from the European Unions Seventh Framework Programme (FP7/2007-2013) under grant agreement No.309589 (M3d). J. A. F.-R. is grateful for financial support from the Spanish MINECO under MAT2016-76824-C3-1-R and co-support from the ESF though BES-2014-068789 and EEBB-I-16-10934. 


\section{References}

[1] http://feellgood.neel.cnrs.fr.

[2] http://scalfmm-public.gforge.inria.fr/doc/.

[3] C. Abraham and A. Aharoni. Linear decrease in the magnetocrystalline anisotropy. Phys. Rev. , 120(5):1576-1579, 1960.

[4] A. Aharoni. Reduction in coercive force caused by a certain type of imperfection. Phys. Rev. , 119(1):127-131, 1960.

[5] S. Allende, D. Altbir, and K. Nielsch. Magnetic cylindrical nanowires with single modulated diameter. Phys. Rev. B , 80(17):174402, 2009.

[6] S. Allende and R. Arias. Transverse domain wall propagation in modulated cylindrical nanostructures and possible geometric control. Phys. Rev. B , 83(17):174452, 2011.

[7] D. A. Allwood, G. Xiong, M. D. Cooke, C. C.Faulkner, D. Atkinson, N. Vernier, and R. P. Cowburn. Submicrometer ferromagnetic not gate and shift register. Science, 296:2003-2006, 2002.

[8] D. A. Allwood, G. Xiong, C. C. Faulkner, D. Atkinson, D. Petit, and R. P. Cowburn. Magnetic domain-wall logic. Science, 309(5741):16881692, 2005.

[9] François Alouges, Evaggelos Kritsikis, Jutta Steiner, and JeanChristophe Toussaint. A convergent and precise finite element scheme for landau-lifschitz-gilbert equation. Numer. Math. , 128(3):407-430, Feb 2014.

[10] S. E. Barnes, J. Ieda, and S. Maekawa. Magnetic memory and current amplification devices using moving domain walls. Appl. Phys. Lett. , 89:122507, 2006.

[11] Uwe Bauer, Satoru Emori, and Geoffrey S. D. Beach. Voltage-controlled domain wall traps in ferromagnetic nanowires. Nat. Nanotech. , 8(6):411-416, may 2013.

[12] R. Becker. Elastic tensions and magnetic characteristics. Phys. ZS, 33:905, 1932. 
[13] R. Becker and W. Döring. Ferromagnetismus. Springer, 1939.

[14] A. Beguivin, L. A. OBrien, A. V. Jausovec, D. Petit, and R. P. Cowburn. Magnetisation reversal in permalloy nanowires controlled by near-field charge interactions. Appl. Phys. Lett. , 99:142506, 2011.

[15] Eider Berganza, Cristina Bran, Manuel Vazquez, and Agustina Asenjo. Domain wall pinning in fecocu bamboo-like nanowires. Sci. Rep. , 6:29702, 2016.

[16] S. Bochmann, D. Döhler, B. Trapp, M. Staňo, A. Wartelle, O. Fruchart, and J. Bachmann. Preparation and physical properties of soft magnetic nickel-cobalt nanowires with modulated diameters. J. Appl. Phys., 124(16):163907, 2018.

[17] S. Bochmann, A. Fernandez-Pacheco, M. Mačkovic̀, A. Neff, K. R. Siefermann, E. Spiecker, R. P. Cowburn, and J. Bachmann. Systematic tuning of segmented magnetic nanowires into three-dimensional arrays of bits. RCS Adv. , 7:37627, 2017.

[18] C Bran, Yu P Ivanov, J Kosel, O Chubykalo-Fesenko, and M Vazquez. $\mathrm{Co} / \mathrm{Au}$ multisegmented nanowires: a $3 \mathrm{~d}$ array of magnetostatically coupled nanopillars. Nanotechnology , 28(9):095709, jan 2017.

[19] W. Brown. Theory of the approach to magnetic saturation. Phys. Rev. $58,1940$.

[20] M. T. Bryan, T. Schrefl, and D. A. Allwood. Symmetric and asymmetric domain wall diodes in magnetic nanowires. Appl. Phys. Lett. , 91(14):142502, oct 2007 .

[21] C. Donnelly, M. Guizar-Sicairos, V. Scagnoli, S. Gliga, M. Holler, J. Raabe, L. Heyderman. Three-dimensional magnetization structures revealed with x-ray vector nanotomography. Nature Vol. 547, 2017.

[22] M. Chandra Sekhar, H. F. Liew, I. Purnama, W. S. Lew, M. Tran, and G. C. Han. Helical domain walls in constricted cylindrical NiFe nanowires. Appl. Phys. Lett. , 101:152406, 2012.

[23] S. Da Col, S. Jamet, M. StaÅo, B. Trapp, S. Le Denmat, L. Cagnon, J. C. Toussaint, and O. Fruchart. Nucleation, imaging, and motion of 
magnetic domain walls in cylindrical nanowires. Applied Physics Letters, 109(6):062406, August 2016.

[24] S. Dubois, L. Piraux, J. M. George, K. Ounadjela, J. L. Duvail, and A. Fert. Evidence for a short spin diffusion length in permalloy from the giant magnetoresistance of multilayered nanowires. Phys. Rev. B, 60(1):477-484, jul 1999.

[25] C. A. Ferguson, D. A. MacLaren, and S. McVitie. Metastable magnetic domain walls in cylindrical nanowires. Journal of Magnetism and Magnetic Materials, 381:457-462, May 2015.

[26] Jose A. Fernandez-Roldan, Rafael P. del Real, Cristina Bran, Manuel Vazquez, and Oksana Chubykalo-Fesenko. Magnetization pinning in modulated nanowires: from topological protection to the "corkscrew" mechanism. Nanoscale, 10:5923, 2018.

[27] A. Fert and J. L. Piraux. Magnetic nanowires. J. Magn. Magn. Mater. , 200:338-358, 1999.

[28] H. Forster, T. Schrefl, W. Scholz, D. Suess, V. Tsiantos, J. Fidler. Micromagnetic simulation of domain wall motion in magnetic nano-wires. Journal of Magnetism and Magnetic Materials 249, 2002.

[29] M. Hayashi, L. Thomas, Ch. Rettner, R. Moriya, and S. S. P. Parkin. Direct observation of the coherent precession of magnetic domain walls propagating along permalloy nanowires. Nat. Phys. , 3(1):21, 2007.

[30] Riccardo Hertel and Attila KÃjkay. Analytic form of transverse headto-head domain walls in thin cylindrical wires. Journal of Magnetism and Magnetic Materials, 379:45-49, April 2015.

[31] Burkard Hillebrands and Andre Thiaville, editors. Spin Dynamics in Confined Magnetic Structures III. Topics in Applied Physics, Spin Dynamics in Confined Magnetic Structures. Springer-Verlag, Berlin Heidelberg, 2006.

[32] Alex Hubert and Rudolf SchÃfer. Magnetic Domains: The Analysis of Magnetic Microstructures. Springer-Verlag, Berlin Heidelberg, 1998. 
[33] Yu P. Ivanov, M. VÃ jzquez, and O. Chubykalo-Fesenko. Magnetic reversal modes in cylindrical nanowires. Journal of Physics D: Applied Physics, 46(48):485001, November 2013.

[34] J. M. D. Coey. Magnetism and Magnetic Materials. Cambridge University Press, 2009.

[35] J.A.Fernandez-Roldan, A.De Riz, B.Trapp, C.Thirion, M.Vazquez, J.C.Toussaint, O.Fruchart and D.Gusakova. Modeling magnetic-fieldinduced domain wall propagation in modulated-diameter cylindrical nanowires. Scientific Report, 9:5130, 2019.

[36] E. Kondorski. On the nature of coercive force and irreversible changes in magnetisation. Phys. Z. Sowjetunion, 11:597, 1937.

[37] E. J. Kondorski. J. Exp. Theor. Fiz., 10:420, 1940.

[38] Benjamin KrÃger. The interaction of transverse domain walls. Journal of Physics: Condensed Matter, 24(2):024209, December 2011.

[39] Reinoud Lavrijsen, Ji-Hyun Lee, Amalio Fernandez-Pacheco, Dorothée C. M. C. Petit, Rhodri Mansell, and Russell P. Cowburn. Magnetic ratchet for three-dimensional spintronic memory and logic. Nature, 493(7434):647-650, 2013.

[40] Woo Lee and Jae-Cheon Kim. Highly ordered porous alumina with tailor-made pore structures fabricated by pulse anodization. Nanotechnology , 21:485304, 2010.

[41] E. R. Lewis, D. Petit, L. OBrien, A. Fernandez-Pacheco, J. Sampaio, A-V. Jausovec, H. T. Zeng D. E. Read, and R. P. Cowburn. Fast domain wall motion in magnetic comb structures. Nat. Mater., page 980, 2010.

[42] M Stano, S Jamet, J C Toussaint, S Bochmann, J Bachmann, A Masseboeuf, C Gatel, O Fruchart. Probing domain walls in cylindrical magnetic nanowires with electron holography. In JEMS-Proceedings, 2016.

[43] Malozemoff, A.P., Slonczewski, J.C. Magnetic Domain Walls in Bubble Materials. Academic Press, 1979. 
[44] N. Biziere, C. Gatel, R. Lassalle-Balier, M. Clochard, J. Wegrowe, E. Snoeck. Imaging the fine structure of a magnetic domain wall in a ni nanocylinder. Nano Lett., 2013.

[45] R. F. Neumann, M. Bahiana, N. M. Vargas, D. Altbir, S. Allende, D. Görlitz, and K. Nielsch. Domain wall control in wire-tube nanoelements. Appl. Phys. Lett., 102:202407, 2013.

[46] Stuart S. P. Parkin, Masamitsu Hayashi, and Luc Thomas. Magnetic domain-wall racetrack memory. Science, 320:190, 2008.

[47] S. Jamet, N. Rougemaille, J.-C. Toussaint, O. Fruchart. Magnetic Nanoand Microwires: Design, synthesis, properties and applications. Woodhead, 2015.

[48] D. Salazar-Aravena, R. M. Corona, D. Goerlitz, and K.Nielsch J. Escrig. Magnetic properties of multisegmented cylindrical nanoparticles with alternating magnetic wire and tube segments. J. Magn. Magn. Mater., 346(0):171 - 174, 2013.

[49] Mohamed Shaker Salem, Philip Sergelius, Rosa M. Corona, Juan Escrig, Detlef Görlitz, and Kornelius Nielsch. Magnetic properties of cylindrical diameter modulated Ni80Fe20 nanowires: interaction and coercive fields. Nanoscale, 2013.

[50] Mohamed Shaker Salem, Felipe Tejo, Robert Zierold, Philip Sergelius, Josep M. Montero Moreno, Detlef Görlitz, Kornelius Nielsch, and Juan Escrig. Composition and diameter modulation of magnetic nanowire arrays fabricated by a novel approach. Nanotechnology , 29:065602, 2018.

[51] J. Sampaio, L. O.'Brien, D. E. Read, E. R. Lewis, H. T. Zeng, D. Petit, R. P. Cowburn anf L. Thevenard, and S. Cardoso. Coupling and induced depinning of magnetic domain walls in adjacent spin valve nanotracks. J. Appl. Phys., 2013.

[52] Luis Serrano-Raman, Amalio Fernandez-Pacheco, Manuel Ricardo Ibarra, Dorothée Petit, Russell P. Cowburn, Tolek Tyliszczak, and José Maria De Teresa. Modification of domain-wall propagation in co nanowires via ga+ irradiation. Europhys. J. B , 86(3):97, Mar 2013. 
[53] C. T. Sousa, D. C. Leitao, M. P. Proenca, J. Ventura, A. M. Pereira, and J. P. Araujo. Nanoporous alumina as templates for multifunctional applications. Appl. Phys. Rev. , 1(3):031102, 2014.

[54] M. Staňo and O. Fruchart. Handbook of Magnetic Materials, volume 27, chapter Magnetic nanowires and nanotubes. Elsevier, 2018.

[55] M. Sturma, J.-C. Toussaint, and D. Gusakova. Geometry effects on magnetization dynamics in circular cross-section wires. J. Appl. Phys., 117(24):243901, jun 2015.

[56] A. Thiaville and Y. Nakatani. Spin dynamics in confined magnetic structures III, volume 101 of Topics Appl. Physics, chapter Domain-wall dynamics in nanowires and nanostrips, pages 161-205. Springer, Berlin, 2006 .

[57] Thiaville, A., Nakatani, Y. Micromagnetic simulation of domain wall dynamics in nanostrips. Nanomagnetism and Spintronics. Elsevier., 2009.

[58] Beatrix Trapp. Nucleation and propagation of magnetic domain walls in cylindrical nanowires with diameter modulations. PhD thesis, 2018.

[59] Andreas Vogel, Sebastian Wintz, Theo Gerhardt, Lars Bocklage, Thomas Strache, Mi-Young Im, Peter Fischer, Jürgen Fassbender, Jeffrey McCord, and Guido Meier. Field- and current-induced domain-wall motion in permalloy nanowires with magnetic soft spots. Appl. Phys. Lett. , 98:202501, 2011.

[60] Y. Yokoyama, Y. Suzuki, S. Yuasa, K. Ando, K. Shigeto, T. Shinjo, P. Gogol, J. Miltat, A. Thiaville, T. Ono, and T. Kawagoe. Kerr microscopy observations of magnetization process in microfabricated ferromagnetic wires. J. Appl. Phys., 87(9):5618, 2000. 